\title{
Exterior Powers and Pointwise Creation Operators
}

\author{
Dimitrios Chiotis $^{1} \cdot$ Zinaida A. Lykova $^{1}$ (C) $\cdot$ N. J. Young ${ }^{1,2}$ (I)
}

Received: 28 April 2020 / Accepted: 14 December 2020 / Published online: 27 January 2021

(c) The Author(s) 2021

\begin{abstract}
We develop a theory of pointwise wedge products of vector-valued functions on the circle and the disc, and obtain results which give rise to a new approach to the analysis of the matricial Nehari problem. We investigate properties of pointwise creation operators and pointwise orthogonal complements in the context of operator theory and the study of vector-valued analytic functions on the unit disc.
\end{abstract}

Keywords Creation operators · Exterior products · Wedge products · Pointwise wedge products

Mathematics Subject Classification 15 A75 $\cdot$ 47B35 $\cdot$ 30J99

\section{Introduction}

The wedge product of Hilbert spaces, though a long established theory (see, for example $[3,5,11,12])$, deserves in our view to be better exploited in operator theory than it

Communicated by Sanne ter Horst.

Chiotis was supported by a Ph.D. studentship from the School of Mathematics, Statistics and Physics of Newcastle University. Lykova and Young were partially supported by the Engineering and Physical Sciences grant EP/N03242X/1. We are very grateful to a referee for numerous improvements of our first version of the paper and for suggestions of missing references.

$\bowtie \quad$ N. J. Young

Nicholas.Young@ncl.ac.uk

Dimitrios Chiotis

chiotisd@gmail.com

Zinaida A. Lykova

Zinaida.Lykova@ncl.ac.uk

1 School of Mathematics, Statistics and Physics, Newcastle University, Newcastle upon Tyne NE1 7RU, UK

2 School of Mathematics, Leeds University, Leeds LS2 9JT, UK

Birkhäuser 
has been hitherto. In this paper we put forward a new approach to some aspects of the analysis of $E$-valued functions on the unit disc $\mathbb{D}$ and on the unit circle $\mathbb{T}$, where $E$ is a separable Hilbert space. Our first application of this idea was to the problem of the superoptimal analytic approximation of a continuous matrix-valued function on the unit circle [2]. The computation of such approximants arises naturally in the context of the classical "Nehari problem", and also in its application to the "robust stabilization problem" in control engineering. In [2] we give a new algorithm for the construction of the unique superoptimal analytic approximant of a continuous matrix-valued function on the unit circle, making use of exterior powers of operators in preference to spectral or Wiener-Masani factorizations. This algorithm is parallel to the construction of [10], but has the advantage that it requires only the spectral factorisation of scalar functions on the circle, together with the calculation of singular-value decompositions, and otherwise requires only rational arithmetic. In the algorithm we make use of pointwise creation operators and pointwise orthogonal complements.

We show how the Hilbert space geometry of the Hardy space $H^{2}(\mathbb{D}, E)$, where $E$ is a separable Hilbert space, interacts with the pointwise geometry of $H^{2}(\mathbb{D}, E)$, that is, the geometry of $E$ over each point of $\mathbb{T}$ or $\mathbb{D}$. We work with the completion of the algebraic tensor product of Hilbert spaces, namely the Hilbert tensor product (see $[4,6])$, and, in particular, with its closed linear subspace of antisymmetric tensors. The space of all antisymmetric tensors $\wedge^{p} E$, also called a wedge or exterior product, is a closed linear subspace of the $p$-fold Hilbert tensor product $\otimes_{H}^{p} E$, see Sect. 2.

In Sect. 3, we define the pointwise wedge product of maps that are defined either on the unit disc or on the unit circle and take values in Hilbert spaces. We illustrate the fact that some classical results from function theory extend to the pointwise wedge product. An exemplary result is Proposition 3.7 which asserts that, for any separable Hilbert space $E$, the pointwise wedge product of two functions in the Hardy space $H^{2}(\mathbb{D}, E)$ is an element of $H^{1}\left(\mathbb{D}, \wedge^{2} E\right)$. We study pointwise creation operators, which are of the form

$$
C_{\xi}: H^{2}(\mathbb{D}, E) \rightarrow H^{2}\left(\mathbb{D}, \wedge^{2} E\right), \quad f \mapsto \xi \dot{\wedge} f
$$

where $\xi: \mathbb{D} \rightarrow E$ is a bounded analytic function, and the symbol $\xi \dot{\wedge} f$ denotes the function

$$
(\xi \dot{\wedge} f)(z)=\xi(z) \wedge f(z) \text { for all } z \in \mathbb{D}
$$

Analogous notation applies when $\xi, f$ are $E$-valued functions defined almost everywhere on $\mathbb{T}$. We connect the kernel of $C_{\xi}$ to the pointwise linear span and pointwise orthogonal complement of $\xi$.

Definition 1.1 Let $E$ be a separable Hilbert space. $H^{\infty}(\mathbb{D}, E)$ denotes the space of bounded analytic $E$-valued functions $Q$ on the unit disk with supremum norm:

$$
\|Q\|_{H^{\infty}} \stackrel{\text { def }}{=}\|Q\|_{\infty} \stackrel{\text { def }}{=} \sup _{z \in \mathbb{D}}\|Q(z)\|_{E} .
$$

In Sect. 4 we prove, among other things, the following two statements. 
Theorem 4.7 Let $E$ be a separable Hilbert space, let $\xi_{0}, \xi_{1}, \ldots, \xi_{j} \in H^{\infty}(\mathbb{D}, E)$. Suppose that the set $\left\{\xi_{i}(z)\right\}_{i=0}^{j}$ is orthonormal in $E$ for almost every $z \in \mathbb{T}$. Then

$$
\xi_{0} \dot{\wedge} \cdots \dot{\wedge} \xi_{j} \dot{\wedge} H^{2}(\mathbb{D}, E)
$$

is a closed subspace of $H^{2}\left(\mathbb{D}, \wedge^{j+2} E\right)$.

Let $F$ and $E$ be Hilbert spaces. The Banach space of bounded linear operators from $F$ to $E$ with the operator norm is denoted by $\mathcal{B}(F, E)$.

Definition 1.2 Let $E$ be a separable Hilbert space and let $\xi, \eta \in L^{\infty}(\mathbb{T}, E)$. We define $\xi \eta^{*} \in L^{\infty}(\mathbb{T}, \mathcal{B}(E, E))$ by

$\left(\xi \eta^{*}\right)(z) x=\langle x, \eta(z)\rangle \xi(z)$ for all $x \in E$ and for almost every $z$ on $\mathbb{T}$.

Thus $\xi \eta^{*}(z)$ is the operator of rank one on $E$ that is sometimes denoted by $\xi(z) \otimes$ $\eta(z)$ (for example, in [1, Eq. (1.17)]).

Theorem 4.18 Let $E$ be a separable Hilbert space. Let $\xi \in H^{\infty}(\mathbb{D}, E)$ be an inner function. Then, for any $h \in H^{2}(\mathbb{D}, E)$,

$$
C_{\xi}^{*} C_{\xi} h=P_{+} \alpha_{\xi},
$$

where $\alpha_{\xi}=h-\xi \xi^{*} h$ and $P_{+}: L^{2}(\mathbb{T}, E) \rightarrow H^{2}(\mathbb{D}, E)$ is the orthogonal projection. Moreover

$$
C_{\xi}^{*} C_{\xi} h=h-T_{\xi \xi} h,
$$

where $T_{\xi \xi^{*}}: H^{2}(\mathbb{D}, E) \rightarrow H^{2}(\mathbb{D}, E)$ is the Toeplitz operator with symbol $\xi \xi^{*}$.

Thus $C_{\xi}^{*} C_{\xi}$ is the Toeplitz operator with symbol $1-\xi \xi^{*}$. Finally, we show that although $C_{\xi}$ is an isometry from the pointwise orthogonal complement of $\xi$ to $H^{2}\left(\mathbb{D}, \wedge^{2} E\right)$, a simple example proves that $C_{\xi}$ fails to be a partial isometry.

\section{Exterior Powers of Hilbert Spaces and Operators}

In this Section we recall the established notion of the exterior power, or wedge product, of Hilbert spaces. One can find definitions and properties of wedge products in $[3,5$, 9,11-13]. Here we present a concise version of this theory which we need for the development of pointwise wedge products of vector-valued functions on the circle and the disc. We shall assume the notion of the algebraic tensor product of linear spaces as given, for example, in [6].

For $E$ a Hilbert space, we consider the completion of the $p$-fold algebraic tensor product, denoted by $\otimes_{H}^{p} E$, with respect to the norm induced by the inner product (2.1). We define an action of permutation operators on tensor products and examine 
various properties. These permutation operators generate two different types of tensors, symmetric and antisymmetric, and we focus on the properties of the antisymmetric tensors. The space of all antisymmetric tensors, also called wedge or exterior products, in $\otimes_{H}^{p} E$ is denoted by $\wedge^{p} E$ and in Theorem 2.9 we prove it is a closed linear subspace of $\otimes_{H}^{p} E$.

In the following $E$ denotes a Hilbert space.

Definition 2.1 $\otimes^{p} E$ is the $p$-fold algebraic tensor product of $E$, spanned by tensors of the form $x_{1} \otimes x_{2} \otimes \cdots \otimes x_{p}$, where $x_{j} \in E$ for $j=1, \ldots, p$.

Definition 2.2 An inner product on $\otimes^{p} E$ is given on elementary tensors by

$$
\left\langle x_{1} \otimes x_{2} \otimes \cdots \otimes x_{p}, y_{1} \otimes y_{2} \otimes \cdots \otimes y_{p}\right\rangle_{\otimes^{p} E}=p !\left\langle x_{1}, y_{1}\right\rangle_{E} \cdots\left\langle x_{p}, y_{p}\right\rangle_{E}
$$

for any $x_{1}, \ldots, x_{p}, y_{1}, \ldots, y_{p} \in E$, and is extended to $\otimes^{p} E$ by sesqui-linearity.

Definition $2.3 \otimes_{H}^{p} E$ is the completion of $\otimes^{p} E$ with respect to the norm $\|u\|=\langle u, u\rangle_{\otimes^{p} E}^{1 / 2}$, for $u \in \otimes^{p} E$.

Observe that the inner product (2.1), in contrast to the majority of the ones included in the bibliography, invokes a multiple of $p$ !. The reason for this choice will be apparent in Theorem 2.11.

In order to introduce antisymmetric tensors, we need to consider the action of the following permutation operators on tensors.

Definition 2.4 Let $\mathfrak{S}_{p}$ denote the symmetric group on $\{1, \ldots, p\}$, with the operation of composition. For $\sigma \in \mathfrak{S}_{p}$, we define

$$
S_{\sigma}: \otimes^{p} E \rightarrow \otimes^{p} E
$$

on elementary tensors by

$$
S_{\sigma}\left(x_{1} \otimes x_{2} \otimes \cdots \otimes x_{p}\right)=x_{\sigma(1)} \otimes x_{\sigma(2)} \otimes \cdots \otimes x_{\sigma(p)},
$$

and we extend $S_{\sigma}$ to $\otimes^{p} E$ by linearity, that is, for $u=\sum_{i=1}^{n} \lambda_{i} x_{1}^{i} \otimes \cdots \otimes x_{p}^{i}$, we define

$$
S_{\sigma}(u)=\sum_{i=1}^{n} \lambda_{i} S_{\sigma}\left(x_{1}^{i} \otimes \cdots \otimes x_{p}^{i}\right) .
$$

for any $x_{j}^{i} \in E$ and $\lambda_{i} \in \mathbb{C}$.

Remark $2.5\left(\mathfrak{S}_{p}, \circ\right)$ is a group, and so, for every permutation $\sigma \in \mathfrak{S}_{p}$, there exists $\sigma^{-1} \in \mathfrak{S}_{p}$ such that

$$
\sigma \circ \sigma^{-1}=\mathrm{id}=\sigma^{-1} \circ \sigma
$$

where id $\in \mathfrak{S}_{p}$ is the identity map on $\{1, \ldots, p\}$. 
Elements of $\mathfrak{S}_{p}$ induce unitary operators on $\otimes_{H}^{p} E$ in an obvious way.

Proposition 2.6 Let E be a Hilbert space, and let $p$ be a positive integer. Then, for any $\sigma \in \mathfrak{S}_{p}, S_{\sigma}$ is a linear operator on the normed space $\left(\otimes^{p} E,\|\cdot\|\right)$, which extends to an isometry $\mathbf{S}_{\sigma}$ on $\left(\otimes_{H}^{p} E,\|\cdot\|\right)$. Furthermore, $\mathbf{S}_{\sigma}$ is a unitary operator on $\otimes_{H}^{p} E$.

Proof It is easy to check that $S_{\sigma}$ is linear. For any elementary tensors $w=x_{1} \otimes x_{2} \otimes$ $\cdots \otimes x_{p}, v=y_{1} \otimes y_{2} \otimes \cdots y_{p}$ by the definition of the inner product on $\otimes^{p} E$,

$$
\begin{aligned}
\left\langle S_{\sigma}^{*} w, v\right\rangle_{\otimes^{p} E} & =\left\langle w, y_{\sigma(1)} \otimes \cdots \otimes y_{\sigma(p)}\right\rangle_{\otimes^{p} E} \\
& =p ! \prod_{j=1}^{p}\left\langle x_{j}, y_{\sigma(j)}\right\rangle_{E} \\
& =p ! \prod_{j=1}^{p}\left\langle x_{\sigma^{-1}(j)}, y_{j}\right\rangle_{E} \\
& =\left\langle S_{\sigma^{-1}} w, v\right\rangle_{\otimes^{p} E} .
\end{aligned}
$$

Hence $S_{\sigma}^{*}=S_{\sigma^{-1}}$, thus

$$
S_{\sigma}^{*} S_{\sigma}=S_{\sigma^{-1}} S_{\sigma}=I
$$

the identity operator on $\otimes^{p} E$, and therefore $S_{\sigma}$ is an isometric linear self-map of $\otimes^{p} E$. Likewise, $S_{\sigma} S_{\sigma}^{*}=I$, and so $S_{\sigma}$ is also a surjective self-map of $\otimes^{p} E$.

Thus one can extend $S_{\sigma}$ by continuity to an isometric linear self-map $\mathbf{S}_{\sigma}$ of the completion $\otimes_{H}^{p} E$ of $\otimes^{p} E$. Since $\mathbf{S}_{\sigma}$ is isometric, its range is complete, hence closed in $\otimes_{H}^{p} E$. Since the range of $\mathbf{S}_{\sigma}$ contains that of $S_{\sigma}, \operatorname{ran} \mathbf{S}_{\sigma}=\otimes_{H}^{p} E$. Being both surjective and isometric, $\mathbf{S}_{\sigma}$ is a unitary operator on $\otimes_{H}^{p} E$.

Henceforth we shall denote the extended operator $\mathbf{S}_{\sigma}$ by $S_{\sigma}$.

Definition 2.7 A tensor $u \in \otimes_{H}^{p} E$ is said to be symmetric if $S_{\sigma}(u)=u$ for all $\sigma \in \mathfrak{S}_{p}$. A tensor $u \in \otimes_{H}^{p} E$ is said to be antisymmetric if $u=\epsilon_{\sigma} S_{\sigma} u$ for all $\sigma \in \mathfrak{S}_{p}$, where $\epsilon_{\sigma}$ is the signature of $\sigma$.

Note that $\epsilon_{\sigma \circ \sigma^{-1}}=\epsilon_{\sigma} \epsilon_{\sigma^{-1}}=1$, and hence $\epsilon_{\sigma}=\epsilon_{\sigma^{-1}}$.

Definition 2.8 The space of all antisymmetric tensors in $\otimes_{H}^{p} E$ will be denoted by $\wedge^{p} E$.

Theorem 2.9 Let $E$ be a Hilbert space. Then $\wedge^{p} E$ is a closed linear subspace of the Hilbert space $\otimes_{H}^{p} E$ for any $p \geq 2$.

Proof For $\sigma \in \mathfrak{S}_{p}$ define the operator

$$
f_{\sigma} \stackrel{\text { def }}{=} S_{\sigma}-\epsilon_{\sigma} I: \otimes_{H}^{p} E \rightarrow \otimes_{H}^{p} E,
$$


where $I$ denotes the identity operator on $\otimes_{H}^{p} E$. Since $S_{\sigma}$ is a continuous linear operator on $\otimes_{H}^{p} E, f_{\sigma}$ is a continuous linear operator. The kernel of the operator $f_{\sigma}$ is

$$
\begin{aligned}
\operatorname{ker} f_{\sigma} & =\left\{u \in \otimes_{H}^{p} E:\left(S_{\sigma}-\epsilon_{\sigma} I\right)(u)=0\right\} \\
& =\left\{u \in \otimes_{H}^{p} E: S_{\sigma}(u)=\epsilon_{\sigma} u\right\} \\
& =\left\{u \in \otimes_{H}^{p} E: \epsilon_{\sigma} S_{\sigma}(u)=u\right\} .
\end{aligned}
$$

Since $f_{\sigma}$ is a continuous linear operator on $\otimes_{H}^{p} E$, ker $f_{\sigma}$ is a closed linear subspace of $\otimes_{H}^{p} E$. Thus $\wedge^{p} E$ is a closed linear subspace of $\otimes_{H}^{p} E$, since

$$
\wedge^{p} E=\left\{u \in \otimes_{H}^{p} E: \epsilon_{\sigma} S_{\sigma}(u)=u \text { for all } \sigma \in \mathfrak{S}_{p}\right\}=\bigcap_{\sigma \in \mathfrak{S}_{p}} \operatorname{ker} f_{\sigma} .
$$

Theorem 2.9 implies that the orthogonal projection from $\otimes_{H}^{p} E$ onto $\wedge^{p} E$ is welldefined.

Definition 2.10 Let $E$ be a Hilbert space. For $x_{1}, \ldots, x_{p} \in E$, define $x_{1} \wedge x_{2} \wedge \cdots \wedge x_{p}$ to be the orthogonal projection of the elementary tensor $x_{1} \otimes x_{2} \otimes \cdots \otimes x_{p}$ onto $\wedge^{p} E$, that is

$$
x_{1} \wedge x_{2} \wedge \cdots \wedge x_{p}=P_{\wedge^{p} E}\left(x_{1} \otimes \cdots \otimes x_{p}\right)
$$

Theorem 2.11 For all $u \in \otimes_{H}^{p} E$,

$$
P_{\wedge^{p} E}(u)=\frac{1}{p !} \sum_{\sigma \in \mathfrak{S}_{p}} \epsilon_{\sigma} S_{\sigma}(u)
$$

Proof Let $u \in \otimes_{H}^{p} E$. Then, for any $\sigma \in \mathfrak{S}_{p}, u=\epsilon_{\sigma} S_{\sigma}(u)+\left(u-\epsilon_{\sigma} S_{\sigma}(u)\right)$, and so

$$
p ! u=\sum_{\sigma \in \mathfrak{S}_{p}} \epsilon_{\sigma} S_{\sigma}(u)+\sum_{\sigma \in \mathfrak{S}_{p}}\left(u-\epsilon_{\sigma} S_{\sigma}(u)\right) .
$$

It suffices to show that $\sum_{\sigma \in \mathfrak{S}_{p}} \epsilon_{\sigma} S_{\sigma}(u) \in \wedge^{p} E$ and

$$
\sum_{\sigma \in \mathfrak{S}_{p}}\left(u-\epsilon_{\sigma} S_{\sigma}(u)\right)
$$

is orthogonal to the set of antisymmetric tensors, in other words, that if $v \in \wedge^{p} E$ then

$$
\left\langle v, \sum_{\sigma \in \mathfrak{S}_{p}}\left(u-\epsilon_{\sigma} S_{\sigma}(u)\right)\right\rangle_{\otimes_{H}^{p} E}=0 .
$$


Let $w=\sum_{\sigma \in \mathfrak{S}_{p}} \epsilon_{\sigma} S_{\sigma}(u) \in \otimes_{H}^{p} E$. For every $\tau \in \mathfrak{S}_{p}$, we have

$$
\begin{aligned}
\epsilon_{\tau} S_{\tau}(w) & =\epsilon_{\tau} S_{\tau}\left(\sum_{\sigma \in \mathfrak{S}_{p}} \epsilon_{\sigma} S_{\sigma}(u)\right)=\sum_{\tau \circ \sigma \in \mathfrak{S}_{p}} \epsilon_{\tau \circ \sigma} S_{\tau \circ \sigma}(u) \\
& =\sum_{\sigma^{\prime} \in \mathfrak{S}_{p}} \epsilon_{\sigma^{\prime}} S_{\sigma^{\prime}}(u)=w
\end{aligned}
$$

where $\tau \circ \sigma=\sigma^{\prime}$. Hence $\sum_{\sigma \in \mathfrak{S}_{p}} \epsilon_{\sigma} S_{\sigma}(u) \in \wedge^{p} E$.

For every $v \in \wedge^{p} E$, we have $v=\epsilon_{\sigma} S_{\sigma} v$ for all $\sigma \in \mathfrak{S}_{p}$, and

$$
\begin{aligned}
\left\langle v, \sum_{\sigma \in \mathfrak{S}_{p}}\left(u-\epsilon_{\sigma} S_{\sigma}(u)\right)\right\rangle_{\otimes_{H}^{p} E} & =\sum_{\sigma \in \mathfrak{S}_{p}}\langle v, u\rangle_{\otimes_{H}^{p} E}-\sum_{\sigma \in \mathfrak{S}_{p}} \epsilon_{\sigma}\left\langle v, S_{\sigma}(u)\right\rangle_{\otimes_{H}^{p} E} \\
& =\sum_{\sigma \in \mathfrak{S}_{p}}\langle v, u\rangle_{\otimes_{H}^{p} E}-\sum_{\sigma \in \mathfrak{S}_{p}} \epsilon_{\sigma}\left\langle S_{\sigma}{ }^{*} v, u\right\rangle_{\otimes_{H}^{p} E} \\
& =\sum_{\sigma \in \mathfrak{S}_{p}}\left\langle v-\epsilon_{\sigma^{-1}} S_{\sigma^{-1}} v, u\right\rangle_{\otimes_{H}^{p} E}=0 .
\end{aligned}
$$

Remark 2.12 If $p>1$, then $\mathfrak{S}_{p}$ contains a transposition, for instance $\sigma=(12)$, and $\epsilon_{\sigma}=-1$. If $p=1$, then $\wedge^{1} E=E$.

Proposition 2.13 Let $E$ be a Hilbert space and let $p \geq 2$. The set of antisymmetric tensors and the set of symmetric tensors are orthogonal in $\otimes_{H}^{p} E$.

Proof Suppose that $u$ is a symmetric tensor, that is $S_{\sigma} u=u$ for all $\sigma \in \mathfrak{S}_{p}$, and that $v$ is an antisymmetric tensor, that is, $S_{\sigma} v=\epsilon_{\sigma} v$ for all $\sigma \in \mathfrak{S}_{p}$. Since $p \geq 2$ there exists a transposition $\sigma \in \mathfrak{S}_{p}$, so that $\epsilon_{\sigma}=-1$, and therefore $S_{\sigma} v=-v$. By Proposition 2.6, $S_{\sigma}$ is a unitary operator on $\otimes_{H}^{p} E$. Thus

$$
\langle u, v\rangle_{\otimes_{H}^{p} E}=\left\langle S_{\sigma} u, S_{\sigma} v\right\rangle_{\otimes_{H}^{p} E}=\langle u,-v\rangle_{\otimes_{H}^{p} E} \cdot
$$

Thus $\langle u, v\rangle_{\otimes_{H}^{p} E}=-\langle u, v\rangle_{\otimes_{H}^{p} E}$, and so $\langle u, v\rangle_{\otimes_{H}^{p} E}=0$.

Proposition 2.14 Let E be a Hilbert space. The inner product in $\wedge^{p} E$ is given by

$$
\left\langle x_{1} \wedge \cdots \wedge x_{p}, y_{1} \wedge \cdots \wedge y_{p}\right\rangle_{\wedge p} E=\operatorname{det}\left(\begin{array}{ccc}
\left\langle x_{1}, y_{1}\right\rangle_{E} & \ldots\left\langle x_{1}, y_{p}\right\rangle_{E} \\
\vdots & \ddots & \vdots \\
\left\langle x_{p}, y_{1}\right\rangle_{E} & \cdots & \left\langle x_{p}, y_{p}\right\rangle_{E}
\end{array}\right)
$$

for all $x_{1}, \ldots, x_{p}, y_{1}, \ldots, y_{p} \in E$. 
Proof By Theorem 2.11, we have

$$
\begin{aligned}
& \left\langle x_{1} \wedge \cdots \wedge x_{p}, y_{1} \wedge \cdots \wedge y_{p}\right\rangle_{\wedge^{p} E} \\
& =\left\langle\frac{1}{p !} \sum_{\sigma \in \mathfrak{S}_{p}} \epsilon_{\sigma} S_{\sigma}\left(x_{1} \otimes x_{2} \otimes \cdots \otimes x_{p}\right), \frac{1}{p !} \sum_{\tau \in \mathfrak{S}_{p}} \epsilon_{\tau} S_{\tau}\left(y_{1} \otimes y_{2} \otimes \cdots \otimes y_{p}\right)\right\rangle_{\otimes_{H}^{p} E} \\
& =\frac{1}{p !^{2}} \sum_{\sigma, \tau \in \mathfrak{S}_{p}}\left\langle\epsilon_{\sigma} S_{\sigma}\left(x_{1} \otimes x_{2} \otimes \cdots \otimes x_{p}\right), \epsilon_{\tau} S_{\tau}\left(y_{1} \otimes y_{2} \otimes \cdots \otimes y_{p}\right)\right\rangle_{\otimes_{H}^{p} E} \\
& =\frac{1}{p !^{2}} \sum_{\sigma, \tau \in \mathfrak{S}_{p}} \epsilon_{\sigma} \epsilon_{\tau}\left\langle x_{1} \otimes x_{2} \otimes \cdots \otimes x_{p}, S_{\sigma}^{*} S_{\tau}\left(y_{1} \otimes y_{2} \otimes \cdots \otimes y_{p}\right)\right\rangle_{\otimes_{H}^{p} E} \\
& =\frac{1}{p !^{2}} \sum_{\sigma, \tau \in \mathfrak{S}_{p}} \epsilon_{\sigma^{-1}} \epsilon_{\tau}\left\langle x_{1} \otimes x_{2} \otimes \cdots \otimes x_{p}, S_{\sigma^{-1}} S_{\tau}\left(y_{1} \otimes y_{2} \otimes \cdots \otimes y_{p}\right)\right\rangle_{\otimes_{H}^{p} E} \\
& =\frac{1}{p !} \sum_{\sigma^{\prime} \in \mathfrak{S}_{p}} \epsilon_{\sigma^{\prime}}\left\langle x_{1} \otimes x_{2} \otimes \cdots \otimes x_{p}, S_{\sigma^{\prime}}\left(y_{1} \otimes y_{2} \otimes \cdots \otimes y_{p}\right)\right\rangle_{\otimes_{H}^{p} E} \\
& =\sum_{\sigma^{\prime} \in \mathfrak{S}_{p}} \epsilon_{\sigma^{\prime}} \prod_{i=1}^{p}\left\langle x_{i}, y_{\sigma^{\prime}(i)}\right\rangle_{E} \\
& =\operatorname{det}\left(\begin{array}{c}
\left\langle x_{1}, y_{1}\right\rangle_{E} \cdots\left\langle x_{1}, y_{p}\right\rangle_{E} \\
\vdots \\
\left\langle x_{p}, y_{1}\right\rangle_{E} \cdots\left\langle x_{p}, y_{p}\right\rangle_{E}
\end{array}\right)
\end{aligned}
$$

by Leibniz' formula.

Since we have already shown that $\wedge^{p} E$ is a closed linear subspace of the Hilbert space $\otimes_{H}^{p} E$, the space $\left(\wedge^{p} E,\langle\cdot, \cdot\rangle_{\wedge^{p}} E\right)$ with inner product given by Proposition 2.14 is itself a Hilbert space.

Lemma 2.15 Suppose $\left\{u_{1}, \ldots, u_{j}\right\}$ is an orthonormal set in $E$. Then, for every $x \in E$,

$$
\left\|u_{1} \wedge \cdots \wedge u_{j} \wedge x\right\|_{\wedge^{j+1} E}=\left\|x-\sum_{i=1}^{j}\left\langle x, u_{i}\right\rangle u_{i}\right\|_{E}
$$

Proof For $x \in E$ we may write

$$
x=x-\sum_{i=1}^{j}\left\langle x, u_{i}\right\rangle u_{i}+\sum_{i=1}^{j}\left\langle x, u_{i}\right\rangle u_{i} .
$$

By Proposition 2.14,

$$
\begin{aligned}
& \left\|u_{1} \wedge \cdots \wedge u_{j} \wedge x\right\|_{\wedge^{j+1} E}^{2} \\
& =\left\langle u_{1} \wedge \cdots \wedge u_{j} \wedge x, u_{1} \wedge \cdots \wedge u_{j} \wedge x\right\rangle_{\wedge^{j+1} E} \\
& =\operatorname{det}\left(\begin{array}{ccccc}
\left\langle u_{1}, u_{1}\right\rangle_{E} & \left\langle u_{1}, u_{2}\right\rangle_{E} & \ldots & \ldots & \left\langle u_{1}, x\right\rangle_{E} \\
\left\langle u_{2}, u_{1}\right\rangle_{E} & \left\langle u_{2}, u_{2}\right\rangle_{E} & \ldots & \ldots & \left\langle u_{2}, x\right\rangle_{E} \\
\vdots & \ldots & \ddots & \ldots & \ldots \\
\left\langle u_{j}, u_{1}\right\rangle_{E} & \left\langle u_{j}, u_{2}\right\rangle_{E} & \ldots & \left\langle u_{j}, u_{j}\right\rangle_{E} & \left\langle u_{j}, x\right\rangle_{E} \\
\left\langle x, u_{1}\right\rangle_{E} & \left\langle x, u_{2}\right\rangle_{E} & \ldots & \ldots & \langle x, x\rangle_{E}
\end{array}\right) .
\end{aligned}
$$


By assumption,

$$
\left\langle u_{i}, u_{k}\right\rangle= \begin{cases}0, & \text { if } i \neq k \\ 1, & \text { if } i=k\end{cases}
$$

and hence

$$
\left\|u_{1} \wedge \cdots \wedge u_{j} \wedge x\right\|_{\wedge^{j+1} E}^{2}=\operatorname{det}\left(\begin{array}{cccc}
1 & 0 & \ldots & \left\langle u_{1}, x\right\rangle_{E} \\
0 & 1 & \ldots & \left\langle u_{2}, x\right\rangle_{E} \\
\vdots & \ddots & \vdots \\
0 & \ldots & 1 & \left\langle u_{j}, x\right\rangle_{E} \\
\left\langle x, u_{1}\right\rangle_{E} & \left\langle x, u_{2}\right\rangle_{E} & \ldots & \langle x, x\rangle_{E}
\end{array}\right)
$$

If, for $k=1, \ldots, j$, we multiply the $k$-th column of the determinant by $\left\langle u_{k}, x\right\rangle_{E}$ and subtract it from the $(j+1)$-th column, we find that

$$
\begin{aligned}
&\left\|u_{1} \wedge \cdots \wedge u_{j} \wedge x\right\|_{\wedge j+1}^{2} E \operatorname{det}\left(\begin{array}{cccc}
1 & 0 & \ldots & 0 \\
0 & 1 & \ldots & 0 \\
\vdots & & \ddots & \vdots \\
0 & \ldots & 1 & 0 \\
\left\langle x, u_{1}\right\rangle_{E}\left\langle x, u_{2}\right\rangle_{E} & \ldots \ldots\langle x, x\rangle_{E}-\sum_{i=1}^{j}\left|\left\langle x, u_{i}\right\rangle_{E}\right|^{2}
\end{array}\right) \\
&=\|x\|_{E}^{2}-\sum_{i=1}^{j}\left|\left\langle x, u_{i}\right\rangle_{E}\right|^{2} \\
&=\left\|x-\sum_{i=1}^{j}\left\langle x, u_{i}\right\rangle_{E} u_{i}\right\|_{E}^{2}
\end{aligned}
$$

the last equality by Pythagoras' theorem.

Definition 2.16 Let $\left(E,\|\cdot\|_{E}\right)$ be a Hilbert space. The $p$-fold Cartesian product of $E$ is defined to be the set

$$
\underbrace{E \times \cdots \times E}_{p \text {-times }}=\left\{\left(x_{1}, \ldots, x_{p}\right): x_{i} \in E\right\} \text {. }
$$

Moreover, we define a norm on $\underbrace{E \times \cdots \times E}_{p-\text { times }}$ by

$$
\left\|\left(x_{1}, \ldots, x_{p}\right)\right\|=\left\{\sum_{i=1}^{p}\left\|x_{i}\right\|_{E}^{2}\right\}^{\frac{1}{2}} .
$$


Definition 2.17 Let $E$ be a Hilbert space. We define the multilinear operator

$$
\Lambda: \underbrace{E \times \cdots \times E}_{p-\text { times }} \rightarrow \wedge^{p} E
$$

by

$$
\Lambda\left(x_{1}, \ldots, x_{p}\right)=x_{1} \wedge \ldots \wedge x_{p} \text { for all } x_{1}, \ldots, x_{p} \in E
$$

Proposition 2.18 [Hadamard's inequality, [7], p. 477] For any matrix

$$
\begin{gathered}
A=\left(a_{i j}\right) \in \mathbb{C}^{n \times n}, \\
|\operatorname{det}(A)| \leq \prod_{j=1}^{n}\left(\sum_{i=1}^{n}\left|a_{i j}\right|^{2}\right)^{1 / 2} \text { and }|\operatorname{det}(A)| \leq \prod_{i=1}^{n}\left(\sum_{j=1}^{n}\left|a_{i j}\right|^{2}\right)^{1 / 2} .
\end{gathered}
$$

Proposition 2.19 Let E be a Hilbert space. Then the multilinear mapping

$$
\Lambda: \underbrace{E \times \cdots \times E}_{p-\text { times }} \rightarrow \wedge^{p} E
$$

is bounded.

Proof Let $x_{i} \in E$ for all $i=1, \ldots, p$. Then $\Lambda\left(x_{1}, \ldots, x_{p}\right)=x_{1} \wedge \ldots \wedge x_{p}$ and

$$
\begin{aligned}
&\left\|\Lambda\left(x_{1}, \ldots, x_{p}\right)\right\|_{\wedge^{p} E}^{2}=\left\|x_{1} \wedge \ldots \wedge x_{p}\right\|_{\wedge^{p} E}^{2} \\
&=\left\langle x_{1} \wedge \ldots \wedge x_{p}, x_{1} \wedge \ldots \wedge x_{p}\right\rangle_{\wedge^{p} E} \\
&=\operatorname{det}\left(\begin{array}{cccc}
\left\langle x_{1}, x_{1}\right\rangle_{E} & \left\langle x_{1}, x_{2}\right\rangle_{E} & \ldots & \left\langle x_{1}, x_{p}\right\rangle_{E} \\
\left\langle x_{2}, x_{1}\right\rangle_{E} & \left\langle x_{2}, x_{2}\right\rangle_{E} & \ldots & \left\langle x_{2}, x_{p}\right\rangle_{E} \\
\vdots & \vdots & \ddots & \ldots \\
\left\langle x_{p}, x_{1}\right\rangle_{E} & \ldots & \ldots & \left.\ldots x_{p}, x_{p}\right\rangle_{E}
\end{array}\right) \geq 0 .
\end{aligned}
$$

Let

$$
X=\left(\begin{array}{cccc}
\left\langle x_{1}, x_{1}\right\rangle_{E} & \left\langle x_{1}, x_{2}\right\rangle_{E} & \ldots & \left\langle x_{1}, x_{p}\right\rangle_{E} \\
\left\langle x_{2}, x_{1}\right\rangle_{E} & \left\langle x_{2}, x_{2}\right\rangle_{E} & \ldots & \left\langle x_{2}, x_{p}\right\rangle_{E} \\
\vdots & \vdots & \ddots & \ldots \\
\left\langle x_{p}, x_{1}\right\rangle_{E} & \ldots & \ldots & \left\langle x_{p}, x_{p}\right\rangle_{E}
\end{array}\right)
$$

By Hadamard's inequality,

$$
|\operatorname{det}(X)| \leq \prod_{j=1}^{p}\left(\sum_{i=1}^{p}\left|\left\langle x_{i}, x_{j}\right\rangle_{E}\right|^{2}\right)^{1 / 2}
$$


Moreover, by the Cauchy-Schwarz inequality,

$$
|\operatorname{det}(X)| \leq \prod_{j=1}^{p}\left\|x_{j}\right\|_{E}\left(\sum_{i=1}^{p}\left\|x_{i}\right\|_{E}^{2}\right)^{1 / 2}
$$

Therefore

$$
\left\|\Lambda\left(x_{1}, \ldots, x_{p}\right)\right\|_{\wedge^{p} E}^{2} \leq \prod_{j=1}^{p}\left\|x_{j}\right\|_{E}\left(\sum_{i=1}^{p}\left\|x_{i}\right\|_{E}^{2}\right)^{1 / 2} .
$$

Let $\left\|\left(x_{1}, \ldots, x_{p}\right)\right\|_{E^{p}} \leq 1$. Since $\left\|x_{j}\right\|_{E} \leq\left\|\left(x_{1}, \ldots, x_{p}\right)\right\|_{E^{p}} \leq 1$ for each $j$, we have

$$
\left\|\Lambda\left(x_{1}, \ldots, x_{p}\right)\right\|_{\wedge^{p} E}^{2} \leq 1
$$

Hence the $p$-linear operator $\Lambda$ is bounded.

\section{Pointwise Wedge Products}

In this section we introduce the notion of pointwise wedge product of vector-valued functions on the unit circle or in the unit disk and explore its features.

Definition 3.1 Let $E$ be a Hilbert space and let $f, g: \mathbb{D} \rightarrow E(f, g: \mathbb{T} \rightarrow E)$ be $E$-valued maps. We define the pointwise wedge product of $f$ and $g$,

$$
f \dot{\wedge} g: \mathbb{D} \rightarrow \wedge^{2} E \quad\left(f \dot{\wedge} g: \mathbb{T} \rightarrow \wedge^{2} E\right)
$$

by

$$
(f \dot{\wedge} g)(z)=f(z) \wedge g(z) \text { for all } z \in \mathbb{D} \quad \text { (for almost all } z \in \mathbb{T}) \text {. }
$$

Definition 3.2 Let $E$ be a Hilbert space and let $\chi_{1}, \ldots, \chi_{n}: \mathbb{D} \rightarrow E\left(\chi_{1}, \ldots, \chi_{n}: \mathbb{T} \rightarrow\right.$ $E$ ) be $E$-valued maps. We call $\chi_{1}, \ldots \chi_{n}$ pointwise linearly dependent on $\mathbb{D}$ (or on $\mathbb{T}$ ) if for all $z \in \mathbb{D}$ (for almost all $z \in \mathbb{T}$ respectively) the vectors $\chi_{1}(z), \ldots, \chi_{n}(z)$ are linearly dependent in $E$.

Remark 3.3 If $x_{1}, \ldots, x_{n}$ are pointwise linearly dependent on $\mathbb{T}$, then

$$
\left(x_{1} \dot{\wedge} \cdots \dot{\wedge} x_{n}\right)(z)=0
$$

for almost all $z \in \mathbb{T}$. 


\subsection{Pointwise Wedge Products on Function Spaces}

For vector-valued $L^{p}$ spaces we use the terminology of [8].

Definition 3.4 Let $E$ be a separable Hilbert space and let $1 \leq p<\infty$. Define

(i) $L^{p}(\mathbb{T}, E)$ to be the normed space of measurable (weakly or strongly, which amounts to the same thing, in view of the separability of $E$ ) $E$-valued maps $f: \mathbb{T} \rightarrow E$ such that

$$
\|f\|_{p}=\left(\frac{1}{2 \pi} \int_{0}^{2 \pi}\left\|f\left(e^{i \theta}\right)\right\|_{E}^{p} d \theta\right)^{1 / p}<\infty
$$

(ii) $H^{p}(\mathbb{D}, E)$ to be the normed space of analytic $E$-valued maps $f: \mathbb{D} \rightarrow E$ such that

$$
\|f\|_{p}=\sup _{0<r<1}\left(\frac{1}{2 \pi} \int_{0}^{2 \pi}\left\|f\left(r e^{i \theta}\right)\right\|_{E}^{p} d \theta\right)^{1 / p}<\infty
$$

(iii) $L^{\infty}(\mathbb{T}, E)$ is the space of essentially bounded measurable $E$-valued functions on the unit circle with essential supremum norm

$$
\|f\|_{L^{\infty}}=\operatorname{ess} \sup _{|z|=1}\|f(z)\|_{E} .
$$

Proposition 3.5 Let $E$ be a separable Hilbert space and let $\frac{1}{p}+\frac{1}{q}=1$, where $1 \leq p, q \leq \infty$. Suppose that $x \in L^{p}(\mathbb{T}, E), y \in L^{q}(\mathbb{T}, E)$. Then

$$
x \dot{\wedge} y \in L^{1}\left(\mathbb{T}, \wedge^{2} E\right)
$$

and

$$
\|x \dot{\wedge} y\|_{L^{1}\left(\mathbb{T}, \wedge^{2} E\right)} \leq\|x\|_{L^{p}(\mathbb{T}, E)}\|y\|_{L^{q}(\mathbb{T}, E)} .
$$

Proof By Proposition 2.14, for all $z \in \mathbb{T}$,

$$
\begin{aligned}
\|(x \dot{\wedge} y)(z)\|_{\wedge^{2} E}^{2} & =\langle x(z) \wedge y(z), x(z) \wedge y(z)\rangle_{\wedge^{2} E} \\
& =\langle x(z), x(z)\rangle_{E} \cdot\langle y(z), y(z)\rangle_{E}-\left|\langle x(z), y(z)\rangle_{E}\right|^{2} \\
& \leq\|x(z)\|_{E}^{2}\|y(z)\|_{E}^{2} .
\end{aligned}
$$

Thus, for all $z \in \mathbb{T}$,

$$
\|(x \dot{\wedge} y)(z)\|_{\wedge^{2} E} \leq\|x(z)\|_{E}\|y(z)\|_{E} .
$$


By Definition 3.4,

$$
\begin{aligned}
\|x \dot{\wedge} y\|_{L^{1}\left(\mathbb{T}, \wedge^{2} E\right)} & =\frac{1}{2 \pi} \int_{0}^{2 \pi}\left\|(x \dot{\wedge} y)\left(e^{i \theta}\right)\right\|_{\wedge^{2} E} d \theta \\
& \leq \frac{1}{2 \pi} \int_{0}^{2 \pi}\left\|x\left(e^{i \theta}\right)\right\|_{E}\left\|y\left(e^{i \theta}\right)\right\|_{E} d \theta .
\end{aligned}
$$

Now, by Hölder's inequality,

$$
\begin{aligned}
& \frac{1}{2 \pi} \int_{0}^{2 \pi}\left\|x\left(e^{i \theta}\right)\right\|_{E}\left\|y\left(e^{i \theta}\right)\right\|_{E} d \theta \\
& \quad \leq\left(\frac{1}{2 \pi} \int_{0}^{2 \pi}\left\|x\left(e^{i \theta}\right)\right\|_{E}^{p} d \theta\right)^{1 / p}\left(\frac{1}{2 \pi} \int_{0}^{2 \pi}\left\|y\left(e^{i \theta}\right)\right\|_{E}^{q} d \theta\right)^{1 / q} .
\end{aligned}
$$

By inequalities (3.2) and (3.3), $x \dot{\wedge} y \in L^{1}\left(\mathbb{T}, \wedge^{2} E\right)$ and the inequality (3.1) holds.

Proposition 3.6 Let $E$ be a Hilbert space and $x_{1}, x_{2}, \ldots, x_{n}: \mathbb{D} \rightarrow E$ be analytic E-valued maps on $\mathbb{D}$. Then,

$$
x_{1} \dot{\wedge} x_{2} \dot{\wedge} \cdots \dot{\wedge} x_{n}: \mathbb{D} \rightarrow \wedge^{n} E
$$

is also analytic on $\mathbb{D}$ and

$$
\begin{aligned}
& \left(x_{1} \dot{\wedge} x_{2} \dot{\wedge} \cdots \dot{\wedge} x_{n}\right)^{\prime}(z) \\
& \quad=x_{1}^{\prime}(z) \wedge x_{2}(z) \wedge \cdots \wedge x_{n}(z)+\cdots+x_{1}(z) \wedge x_{2}(z) \wedge \cdots \wedge x_{n}^{\prime}(z)
\end{aligned}
$$

for all $z \in \mathbb{D}$.

The proof is straightforward. It follows from Proposition 2.14 and the continuity of $\Lambda$, see Hadamard's inequalities (2.2).

Proposition 3.7 Let E be a separable Hilbert space. Suppose $x, y \in H^{2}(\mathbb{D}, E)$. Then

$$
x \dot{\wedge} y \in H^{1}\left(\mathbb{D}, \wedge^{2} E\right) .
$$

Proof By Proposition 3.6, $x \dot{\wedge} y$ is analytic on $\mathbb{D}$. By Proposition 2.14, for $0<r<1$ and $0 \leq \theta \leq 2 \pi$,

$$
\left\|(x \dot{\wedge} y)\left(r e^{i \theta}\right)\right\|_{\wedge^{2} E} \leq\left\|x\left(r e^{i \theta}\right)\right\|_{E}\left\|y\left(r e^{i \theta}\right)\right\|_{E} .
$$


By Proposition 2.14 and by Definition 3.4,

$$
\begin{aligned}
\|x \dot{\wedge} y\|_{H^{1}\left(\mathbb{D}, \wedge^{2} E\right)} & =\sup _{0<r<1}\left(\frac{1}{2 \pi} \int_{0}^{2 \pi}\left\|(x \dot{\wedge} y)\left(r e^{i \theta}\right)\right\|_{\wedge^{2} E} d \theta\right) \\
& \leq \sup _{0<r<1}\left(\frac{1}{2 \pi} \int_{0}^{2 \pi}\left\|x\left(r e^{i \theta}\right)\right\|_{E}\left\|y\left(r e^{i \theta}\right)\right\|_{E} d \theta\right),
\end{aligned}
$$

for $0<r<1$ and $0 \leq \theta \leq 2 \pi$. Also, by Hölder's inequality, for $0<r<1$ and $0 \leq \theta \leq 2 \pi$,

$$
\begin{aligned}
& \frac{1}{2 \pi} \int_{0}^{2 \pi}\left\|x\left(r e^{i \theta}\right)\right\|_{E}\left\|y\left(r e^{i \theta}\right)\right\|_{E} d \theta \\
& \leq\left(\frac{1}{2 \pi} \int_{0}^{2 \pi}\left\|x\left(r e^{i \theta}\right)\right\|_{E}^{2} d \theta\right)^{1 / 2}\left(\frac{1}{2 \pi} \int_{0}^{2 \pi}\left\|y\left(r e^{i \theta}\right)\right\|_{E}^{2} d \theta\right)^{1 / 2}
\end{aligned}
$$

hence

$$
\|x \dot{\wedge} y\|_{H^{1}\left(\mathbb{D}, \wedge^{2} E\right)} \leq\|x\|_{H^{2}(\mathbb{D}, E)}\|y\|_{H^{2}(\mathbb{D}, E)}
$$

Consequently, $x \dot{\wedge} y \in H^{1}\left(\mathbb{D}, \wedge^{2} E\right)$.

Proposition 3.8 Let $E$ be a separable Hilbert space, let $x \in H^{2}(\mathbb{D}, E)$ and let $y \in$ $H^{\infty}(\mathbb{D}, E)$. Then

$$
x \dot{\wedge} y \in H^{2}\left(\mathbb{D}, \wedge^{2} E\right) .
$$

Proof By Proposition 3.6, $x \dot{\wedge} y$ is analytic on $\mathbb{D}$. By Proposition 3.5, for

$$
0<r<1, \quad 0 \leq \theta \leq 2 \pi
$$

we have

$$
\left\|(x \dot{\wedge} y)\left(r e^{i \theta}\right)\right\|_{\wedge^{2} E} \leq\left\|x\left(r e^{i \theta}\right)\right\|_{E}\left\|y\left(r e^{i \theta}\right)\right\|_{E} .
$$

Thus,

$$
\begin{aligned}
\|x \dot{\wedge} y\|_{H^{2}\left(\mathbb{D}, \wedge^{2} E\right)} & =\sup _{0<r<1}\left(\frac{1}{2 \pi} \int_{0}^{2 \pi}\left\|(x \dot{\wedge} y)\left(r e^{i \theta}\right)\right\|_{\wedge^{2} E}^{2} d \theta\right)^{1 / 2} \\
& \leq \sup _{0<r<1}\left(\frac{1}{2 \pi} \int_{0}^{2 \pi}\left\|x\left(r e^{i \theta}\right)\right\|_{E}^{2}\left\|y\left(r e^{i \theta}\right)\right\|_{E}^{2} d \theta\right)^{1 / 2} \\
& \leq\|y\|_{\infty} \sup _{0<r<1}\left(\frac{1}{2 \pi} \int_{0}^{2 \pi}\left\|x\left(r e^{i \theta}\right)\right\|_{E}^{2} d \theta\right)^{1 / 2}<\infty .
\end{aligned}
$$


Definition 3.9 Let $E$ be a Hilbert space. We say that a family of $\left\{f_{\lambda}\right\}_{\lambda \in \Lambda}$ of maps from $\mathbb{T}$ to $E$ is pointwise orthonormal on $\mathbb{T}$, if for all $z$ in a set of full measure in $\mathbb{T}$, the set of vectors $\left\{f_{\lambda}(z)\right\}_{\lambda \in \Lambda}$ is orthonormal in $E$.

Definition 3.10 Let $E$ be a separable Hilbert space. Let $F$ be a subspace of $L^{2}(\mathbb{T}, E)$ and let $X$ be a subset of $L^{2}(\mathbb{T}, E)$. We define the pointwise orthogonal complement of $X$ in $F$ to be the set

$$
\operatorname{POC}(X, F)=\{f \in F: f(z) \perp\{x(z): x \in X\} \text { for almost all } z \in \mathbb{T}\} .
$$

Proposition 3.11 Let $E$ be a separable Hilbert space, and let $\xi_{0}, \xi_{1}, \ldots, \xi_{j} \in$ $L^{\infty}(\mathbb{T}, E)$ be a pointwise orthonormal set on $\mathbb{T}$, and let $x \in L^{2}(\mathbb{T}, E)$. Then

$$
\xi_{0} \dot{\wedge} \xi_{1} \dot{\wedge} \cdots \dot{\wedge} \xi_{j} \dot{\wedge} x \in L^{2}\left(\mathbb{T}, \wedge^{j+2} E\right),
$$

and

$$
\left\|\xi_{0} \dot{\wedge} \xi_{1} \dot{\wedge} \cdots \dot{\wedge} \xi_{j} \dot{\wedge} x\right\|_{L^{2}(\mathbb{T}, \wedge j+2 E)} \leq\|x\|_{L^{2}(\mathbb{T}, E)}
$$

Furthemore,

$$
\left\|\xi_{0} \dot{\wedge} \xi_{1} \dot{\wedge} \cdots \dot{\wedge} \xi_{j} \dot{\wedge} x\right\|_{L^{2}\left(\mathbb{T}, \wedge^{j+2} E\right)}=\|x\|_{L^{2}(\mathbb{T}, E)}
$$

if and only if $x \in \operatorname{POC}\left(\left\{\xi_{0}, \xi_{1}, \ldots, \xi_{j}\right\}, L^{2}(\mathbb{T}, E)\right)$.

Proof By Lemma 2.15, for almost all $z \in \mathbb{T}$,

$$
\begin{aligned}
& \left\|\xi_{0}(z) \wedge \xi_{1}(z) \wedge \cdots \wedge \xi_{j}(z) \wedge x(z)\right\|_{\wedge j+2}^{2} E \\
& \quad=\|x(z)\|_{E}^{2}-\sum_{i=0}^{j}\left|\left\langle x(z), \xi_{i}(z)\right\rangle_{E}\right|^{2} \leq\|x(z)\|_{E}^{2} .
\end{aligned}
$$

Thus,

$$
\begin{aligned}
& \left\|\xi_{0} \dot{\wedge} \xi_{1} \dot{\wedge} \cdots \dot{\wedge} \xi_{j} \dot{\wedge} x\right\|_{L^{2}\left(\mathbb{T}, \wedge^{j+2} E\right)} \\
& \quad=\left(\frac{1}{2 \pi} \int_{0}^{2 \pi}\left\|\xi_{0}\left(e^{i \theta}\right) \wedge \xi_{1}\left(e^{i \theta}\right) \wedge \cdots \wedge \xi_{j}\left(e^{i \theta}\right) \wedge x\left(e^{i \theta}\right)\right\|_{\wedge^{j+2} E}^{2} d \theta\right)^{1 / 2} \\
& \quad \leq\left(\frac{1}{2 \pi} \int_{0}^{2 \pi}\left\|x\left(e^{i \theta}\right)\right\|_{E}^{2} d \theta\right)^{1 / 2}=\|x\|_{L^{2}(\mathbb{T}, E)}<\infty
\end{aligned}
$$




\section{Pointwise Creation Operators, Orthogonal Complements and Linear Spans}

Definition 4.1 Let $E$ be a separable Hilbert space. Let $\xi \in H^{\infty}(\mathbb{D}, E)$. We define the pointwise creation operator

$$
C_{\xi}: H^{2}(\mathbb{D}, E) \rightarrow H^{2}\left(\mathbb{D}, \wedge^{2} E\right)
$$

by

$$
C_{\xi} f=\xi \dot{\wedge} f, \quad \text { for } f \in H^{2}(\mathbb{D}, E) .
$$

Remark 4.2 Let $E$ be a separable Hilbert space. Let $\xi \in H^{\infty}(\mathbb{D}, E)$ and let $f \in$ $H^{2}(\mathbb{D}, E)$. By the generalized Fatou's Theorem [8, Chapter V], the radial limits

$$
\lim _{r \rightarrow 1} \xi\left(r e^{i \theta}\right) \underset{\|\cdot\|_{E}}{=} \tilde{\xi}\left(e^{i \theta}\right), \quad \lim _{r \rightarrow 1} f\left(r e^{i \theta}\right) \underset{\|\cdot\|_{E}}{=} \tilde{f}\left(e^{i \theta}\right)(0<r<1)
$$

exist almost everywhere on $\mathbb{T}$ and define functions $\tilde{\xi} \in L^{\infty}(\mathbb{T}, E)$ and $\tilde{f} \in L^{2}(\mathbb{T}, E)$ respectively, which satisfy the relations

$$
\lim _{r \rightarrow 1}\left\|\xi\left(r e^{i \theta}\right)-\tilde{\xi}\left(e^{i \theta}\right)\right\|_{E}=0, \quad \lim _{r \rightarrow 1}\left\|f\left(r e^{i \theta}\right)-\tilde{f}\left(e^{i \theta}\right)\right\|_{E}=0 \quad(0<r<1)
$$

for almost all $e^{i \theta} \in \mathbb{T}$.

Lemma 4.3 Let $E$ be a separable Hilbert space. Let $\xi \in H^{\infty}(\mathbb{D}, E)$ and let $f \in$ $H^{2}(\mathbb{D}, E)$. Then the radial limits $\lim _{r \rightarrow 1}\left(\xi\left(r e^{i \theta}\right) \wedge f\left(r e^{i \theta}\right)\right)$ exist for almost all $e^{i \theta} \in$ $\mathbb{T}$ and define functions in $L^{2}\left(\mathbb{T}, \wedge^{2} E\right)$.

Proof By Proposition 2.19, the bilinear operator $\Lambda: E \times E \rightarrow \wedge^{2} E$ is a continuous operator for the norms of $E$ and $\wedge^{2} E$. By Remark 4.2, the functions $\xi \in H^{\infty}(\mathbb{D}, E)$ and $f \in H^{2}(\mathbb{D}, E)$ have radial limit functions $\tilde{\xi} \in L^{\infty}(\mathbb{T}, E)$ and $\tilde{f} \in L^{2}(\mathbb{T}, E)$. Also, by Proposition 3.8, $\xi \dot{\wedge} f \in H^{2}\left(\mathbb{D}, \wedge^{2} E\right)$. Hence

$$
\lim _{r \rightarrow 1}\left\|\xi\left(r e^{i \theta}\right) \wedge f\left(r e^{i \theta}\right)-\tilde{\xi}\left(e^{i \theta}\right) \wedge \tilde{f}\left(e^{i \theta}\right)\right\|_{\wedge^{2} E}=0 \quad \text { almost everywhere on } \mathbb{T}
$$

and we conclude that

$$
\lim _{r \rightarrow 1}\left(\xi\left(r e^{i \theta}\right) \wedge f\left(r e^{i \theta}\right)\right) \underset{\|\cdot\|_{\wedge^{2} E}}{=} \tilde{\xi}\left(e^{i \theta}\right) \wedge \tilde{f}\left(e^{i \theta}\right) \text { almost everywhere on } \mathbb{T} .
$$

This shows that the radial limits

$$
\lim _{r \rightarrow 1}\left(\xi\left(r e^{i \theta}\right) \wedge f\left(r e^{i \theta}\right)\right)
$$


exist almost everywhere on $\mathbb{T}$ and, by Lemma 3.5, define functions in $L^{2}\left(\mathbb{T}, \wedge^{2} E\right)$. Hence one can consider $\left(C_{\xi} f\right)(z)=(\xi \dot{\wedge} f)(z)$ to be defined for either all $z \in \mathbb{D}$ or for almost all $z \in \mathbb{T}$.

Remark 4.4 Let $E$ be a separable Hilbert space. By [8, Chapter 5, Sect. 1], for any separable Hilbert space $E$, the map $f \mapsto \tilde{f}$ is an isometric embedding of $H^{2}(\mathbb{D}, E)$ in $L^{2}(\mathbb{T}, E)$, where $\tilde{f}\left(e^{i \theta}\right)=\lim _{r \rightarrow 1} f\left(r e^{i \theta}\right)$. Since $H^{2}(\mathbb{D}, E)$ is complete and the embedding is isometric, the image of the embedding is complete, and therefore is closed in $L^{2}(\mathbb{T}, E)$. Therefore, the space $H^{2}(\mathbb{D}, E)$ is identified isometrically with a closed linear subspace of $L^{2}(\mathbb{T}, E)$. In future we shall use the same notation for $f$ and $\tilde{f}$.

Our next aim is to show that $\operatorname{POC}(X, F)$ is a closed subspace of $F$. We are going to need the following results.

Lemma 4.5 Let $E$ be a Hilbert space and let $x \in L^{2}(\mathbb{T}, E)$. The function $\varphi: L^{2}(\mathbb{T}, E) \rightarrow \mathbb{C}$ given by

$$
\varphi(g)=\frac{1}{2 \pi} \int_{0}^{2 \pi}\left|\left\langle g\left(e^{i \theta}\right), x\left(e^{i \theta}\right)\right\rangle_{E}\right| d \theta
$$

is continuous.

Proof Consider $g_{0} \in L^{2}(\mathbb{T}, E)$. For any $\epsilon>0$, we are looking for a $\delta>0$ such that

$$
\left\|g-g_{0}\right\|_{L^{2}(\mathbb{T}, E)}=\left(\frac{1}{2 \pi} \int_{0}^{2 \pi}\left\|g\left(e^{i \theta}\right)-g_{0}\left(e^{i \theta}\right)\right\|_{E}^{2} d \theta\right)^{1 / 2}<\delta
$$

implies

$$
\left|\varphi(g)-\varphi\left(g_{0}\right)\right|<\epsilon
$$

Note that

$$
\begin{aligned}
\left|\varphi(g)-\varphi\left(g_{0}\right)\right| & =\left|\frac{1}{2 \pi} \int_{0}^{2 \pi}\right|\left\langle g\left(e^{i \theta}\right), x\left(e^{i \theta}\right)\right\rangle_{E}\left|d \theta-\frac{1}{2 \pi} \int_{0}^{2 \pi}\right|\left\langle g_{0}\left(e^{i \theta}\right), x\left(e^{i \theta}\right)\right\rangle_{E}|d \theta| \\
& =\left|\frac{1}{2 \pi} \int_{0}^{2 \pi}\left(\left|\left\langle g\left(e^{i \theta}\right), x\left(e^{i \theta}\right)\right\rangle_{E}\right|-\left|\left\langle g_{0}\left(e^{i \theta}\right), x\left(e^{i \theta}\right)\right\rangle_{E}\right|\right) d \theta\right|
\end{aligned}
$$

For each $e^{i \theta} \in \mathbb{T}$, by the reverse triangle inequality, the integrand satisfies

$$
\begin{aligned}
\left|\left\langle g\left(e^{i \theta}\right), x\left(e^{i \theta}\right)\right\rangle_{E}\right|-\left|\left\langle g_{0}\left(e^{i \theta}\right), x\left(e^{i \theta}\right)\right\rangle_{E}\right| & \leq\left|\left\langle g\left(e^{i \theta}\right), x\left(e^{i \theta}\right)\right\rangle_{E}-\left\langle g_{0}\left(e^{i \theta}\right), x\left(e^{i \theta}\right)\right\rangle_{E}\right| \\
& =\left|\left\langle\left(g e^{i \theta}\right)-g_{0}\left(e^{i \theta}\right), x\left(e^{i \theta}\right)\right\rangle_{E}\right|,
\end{aligned}
$$




$$
\left|\varphi(g)-\varphi\left(g_{0}\right)\right| \leq \frac{1}{2 \pi} \int_{0}^{2 \pi}\left|\left\langle g\left(e^{i \theta}\right)-g_{0}\left(e^{i \theta}\right), x\left(e^{i \theta}\right)\right\rangle_{E}\right| d \theta .
$$

By the Cauchy-Schwarz inequality,

$$
\begin{aligned}
& \frac{1}{2 \pi} \int_{0}^{2 \pi}\left|\left\langle g\left(e^{i \theta}\right)-g_{0}\left(e^{i \theta}\right), x\left(e^{i \theta}\right)\right\rangle_{E}\right| d \theta \\
& \leq\left(\frac{1}{2 \pi} \int_{0}^{2 \pi}\left\|g\left(e^{i \theta}\right)-g_{0}\left(e^{i \theta}\right)\right\|_{E}^{2} d \theta\right)^{1 / 2}\left(\frac{1}{2 \pi} \int_{0}^{2 \pi}\left\|x\left(e^{i \theta}\right)\right\|_{E}^{2} d \theta\right)^{1 / 2} .
\end{aligned}
$$

For the given $\epsilon>0$, let $\delta$ be equal to $\frac{\epsilon}{\|x\|_{L^{2}(\mathbb{T}, E)}+1}$, and let

$$
\left(\frac{1}{2 \pi} \int_{0}^{2 \pi}\left\|g\left(e^{i \theta}\right)-g_{0}\left(e^{i \theta}\right)\right\|_{E}^{2} d \theta\right)^{1 / 2}<\delta .
$$

By Eqs. (4.1) and (4.2),

$$
\begin{aligned}
\left|\varphi(g)-\varphi\left(g_{0}\right)\right| & \leq\left(\frac{1}{2 \pi} \int_{0}^{2 \pi}\left\|g\left(e^{i \theta}\right)-g_{0}\left(e^{i \theta}\right)\right\|_{E}^{2} d \theta\right)^{1 / 2}\|x\|_{L^{2}(\mathbb{T}, E)} \\
& <\frac{\epsilon}{\|x\|_{L^{2}(\mathbb{T}, E)}+1}\|x\|_{L^{2}(\mathbb{T}, E)}<\epsilon .
\end{aligned}
$$

Hence $\varphi$ is a continuous function.

Proposition 4.6 Let $E$ be a separable Hilbert space. Let $\eta \in L^{2}(\mathbb{D}, E)$. Then

(i) The space $V=\left\{f \in H^{2}(\mathbb{D}, E):\langle f(z), \eta(z)\rangle_{E}=0\right.$ for almost all $\left.z \in \mathbb{T}\right\}$ is a closed subspace of $H^{2}(\mathbb{D}, E)$.

(ii) The space $V=\left\{f \in L^{2}(\mathbb{T}, E):\langle f(z), \eta(z)\rangle_{E}=0\right.$ for almost all $\left.z \in \mathbb{T}\right\}$ is a closed subspace of $L^{2}(\mathbb{T}, E)$.

Proof (i). $V$ is a linear subspace of $H^{2}(\mathbb{D}, E)$ since for $\lambda, \mu \in \mathbb{C}, \psi, k \in V$ and for almost all $z \in \mathbb{T}$,

$$
\langle\lambda \psi(z)+\mu k(z), \eta(z)\rangle_{E}=\lambda\langle\psi(z), \eta(z)\rangle_{E}+\mu\langle k(z), \eta(z)\rangle_{E}=0,
$$

hence $\lambda \psi+\mu k \in V$. 
Now suppose that the sequence of functions $\left(g_{n}\right)_{n=1}^{\infty}$ in $V$ converges to a function $g$. We need to show that $g \in V$. Since $g_{n} \in V$ for all $n \in \mathbb{N}$, we have

$$
\left\langle g_{n}(z), \eta(z)\right\rangle_{E}=0 \text { for almost all } z \in \mathbb{T} \text {. }
$$

Consider the function $\varphi: H^{2}(\mathbb{D}, E) \rightarrow \mathbb{C}$ given by

$$
\varphi(f)=\frac{1}{2 \pi} \int_{0}^{2 \pi}\left|\left\langle f\left(e^{i \theta}\right), \eta\left(e^{i \theta}\right)\right\rangle_{E}\right| d \theta .
$$

Then, by Eq. (4.3), we have

$$
\varphi\left(g_{n}\right)=\frac{1}{2 \pi} \int_{0}^{2 \pi}\left|\left\langle g_{n}\left(e^{i \theta}\right), \eta\left(e^{i \theta}\right)\right\rangle_{E}\right| d \theta=0 .
$$

By Remark 4.4 and Lemma $4.5, \varphi$ is a continuous function on $H^{2}(\mathbb{D}, E)$, thus

$$
\lim _{n \rightarrow \infty} \varphi\left(g_{n}\right)=\varphi(g)
$$

and so

$$
\frac{1}{2 \pi} \int_{0}^{2 \pi}\left|\left\langle g\left(e^{i \theta}\right), \eta\left(e^{i \theta}\right)\right\rangle_{E}\right| d \theta=\lim _{n \rightarrow \infty} \frac{1}{2 \pi} \int_{0}^{2 \pi}\left|\left\langle g_{n}\left(e^{i \theta}\right), \eta\left(e^{i \theta}\right)\right\rangle_{E}\right| d \theta=0 .
$$

Thus $\left|\left\langle g\left(e^{i \theta}\right), \eta\left(e^{i \theta}\right)\right\rangle_{E}\right|=0$ for almost all $e^{i \theta} \in \mathbb{T}$, and, hence, $g \in V$. We have proved that $V$ is a closed subspace of $H^{2}(\mathbb{D}, E)$.

(ii). The proof is similar to (i).

Our motivation for the next theorem is the following. In [2] spaces of the form

$$
\xi_{0} \dot{\wedge} \cdots \dot{\wedge} \xi_{j} \dot{\wedge} H^{2}(\mathbb{D}, E)
$$

play a crucial role in the superoptimal Nehari problem. They are the domains of Hankeltype operators whose norms are "superoptimal singular values" of error functions $G-$ $Q$, where $G$ is a given continuous approximand and $Q$ is its analytic approximation.

Theorem 4.7 Let $E$ be a separable Hilbert space, let $\xi_{0}, \xi_{1}, \ldots, \xi_{j} \in H^{\infty}(\mathbb{D}, E)$. Suppose that the set $\left\{\xi_{i}(z)\right\}_{i=0}^{j}$ is orthonormal in $E$ for almost every $z \in \mathbb{T}$. Then

$$
\xi_{0} \dot{\wedge} \cdots \dot{\wedge} \xi_{j} \dot{\wedge} H^{2}(\mathbb{D}, E)
$$

is a closed subspace of $H^{2}\left(\mathbb{D}, \wedge^{j+2} E\right)$. 
Proof By Proposition 3.6, for every $x \in H^{2}(\mathbb{D}, E)$,

$$
\xi_{0} \dot{\wedge} \xi_{1} \dot{\wedge} \cdots \dot{\wedge} \xi_{j} \dot{\wedge} x
$$

is analytic on $\mathbb{D}$. By Proposition 3.11 , since $\xi_{0}, \xi_{1}, \ldots, \xi_{j}$ are pointwise orthogonal on $\mathbb{T}$,

$$
\left\|\xi_{0} \dot{\wedge} \xi_{1} \dot{\wedge} \cdots \xi_{j} \dot{\wedge} x\right\|_{L^{2}(\mathbb{T}, \wedge j+2 E)}<\infty
$$

Thus, for every $x \in H^{2}(\mathbb{D}, E)$,

$$
\xi_{0} \dot{\wedge} \xi_{1} \dot{\wedge} \cdots \dot{\wedge} \xi_{j} \dot{\wedge} x \in H^{2}\left(\mathbb{D}, \wedge^{j+2} E\right)
$$

Let us first show that $\xi_{0} \dot{\wedge} H^{2}(\mathbb{D}, E)$ is a closed subspace of $H^{2}\left(\mathbb{D}, \wedge^{2} E\right)$. Observe that, by Proposition $4.3, \xi_{0} \dot{\wedge} H^{2}(\mathbb{D}, E) \subset H^{2}\left(\mathbb{D}, \wedge^{2} E\right)$. Let

$$
\Xi_{0}=\left\{f \in H^{2}(\mathbb{D}, E):\left\langle f(z), \xi_{0}(z)\right\rangle_{E}=0 \text { almost everywhere on } \mathbb{T}\right\} .
$$

Consider a vector-valued function $w \in H^{2}(\mathbb{D}, E)$. For almost every $z \in \mathbb{T}$, we may write $w$ as

$$
w(z)=w(z)-\left\langle w(z), \xi_{0}(z)\right\rangle_{E} \xi_{0}(z)+\left\langle w(z), \xi_{0}(z)\right\rangle_{E} \xi_{0}(z)
$$

Then, for all $w \in H^{2}(\mathbb{D}, E)$ and for almost every $z \in \mathbb{T}$,

$$
\begin{aligned}
\left(\xi_{0} \dot{\wedge} w\right)(z) & =\xi_{0}(z) \wedge\left(w(z)-\left\langle w(z), \xi_{0}(z)\right\rangle_{E} \xi_{0}(z)+\left\langle w(z), \xi_{0}(z)\right\rangle_{E} \xi_{0}(z)\right) \\
& =\xi_{0}(z) \wedge\left(w(z)-\left\langle w(z), \xi_{0}(z)\right\rangle_{E} \xi_{0}(z)\right)
\end{aligned}
$$

due to the pointwise linear dependence of $\xi_{0}$ and $z \mapsto\left\langle w(z), \xi_{0}(z)\right\rangle_{E} \xi_{0}(z)$ almost everywhere on $\mathbb{T}$. Note that

$$
w(z)-\left\langle w(z), \xi_{0}(z)\right\rangle_{E} \xi_{0}(z) \in \Xi_{0}
$$

thus

$$
\xi_{0} \dot{\wedge} H^{2}(\mathbb{D}, E) \subset \xi_{0} \dot{\wedge} \Xi_{0}
$$

By Proposition 4.6, $\Xi_{0}$ is a closed subspace of $H^{2}(\mathbb{D}, E)$, hence

$$
\xi_{0} \dot{\wedge} H^{2}(\mathbb{D}, E) \supset \xi_{0} \dot{\wedge} \Xi_{0},
$$

and so,

$$
\xi_{0} \dot{\wedge} H^{2}(\mathbb{D}, E)=\xi_{0} \dot{\wedge} \Xi_{0} .
$$


Consider the mapping

$$
C_{\xi_{0}}: \Xi_{0} \rightarrow \xi_{0} \dot{\wedge} \Xi_{0}
$$

given by

$$
C_{\xi_{0}} w=\xi_{0} \dot{\wedge} w
$$

for all $w \in \Xi_{0}$. Notice that, by assumption, $\left\|\xi_{0}\left(e^{i \theta}\right)\right\|_{E}^{2}=1$ for almost every $e^{i \theta} \in \mathbb{T}$. Therefore, for any $w \in \Xi_{0}$, we have

$$
\begin{aligned}
\left\|\xi_{0} \dot{\wedge} w\right\|_{L^{2}\left(\mathbb{T}, \wedge^{2} E\right)}^{2} & =\frac{1}{2 \pi} \int_{0}^{2 \pi}\left\langle\xi_{0} \dot{\wedge} w, \xi_{0} \dot{\wedge} w\right\rangle\left(e^{i \theta}\right) d \theta \\
& =\frac{1}{2 \pi} \int_{0}^{2 \pi}\left(\left\|\xi_{0}\left(e^{i \theta}\right)\right\|_{E}^{2}\left\|w\left(e^{i \theta}\right)\right\|_{E}^{2}-\left|\left\langle w\left(e^{i \theta}\right), \xi_{0}\left(e^{i \theta}\right)\right\rangle\right|^{2}\right) d \theta \\
& =\|w\|_{L^{2}(\mathbb{T}, E)}^{2},
\end{aligned}
$$

since $w$ is pointwise orthogonal to $\xi_{0}$ almost everywhere on $\mathbb{T}$. Thus the mapping

$$
C_{\xi_{0}}: \Xi_{0} \rightarrow \xi_{0} \dot{\wedge} \Xi_{0}
$$

is an isometry. Furthermore, $C_{\xi_{0}}: \Xi_{0} \rightarrow \xi_{0} \dot{\wedge} \Xi_{0}$ is a surjective mapping, thus $\Xi_{0}$ and $\xi_{0} \dot{\wedge} \Xi_{0}$ are isometrically isomorphic. Therefore, since $\Xi_{0}$ is a closed subspace of $H^{2}(\mathbb{D}, E)$, the space $\xi_{0} \dot{\wedge} \Xi_{0}$ is a closed subspace of $H^{2}\left(\mathbb{D}, \wedge^{2} E\right)$. Hence $\xi_{0} \dot{\wedge} H^{2}(\mathbb{D}, E)$ is a closed subspace of $H^{2}\left(\mathbb{D}, \wedge^{2} E\right)$.

To prove that $\xi_{0} \dot{\wedge} \cdots \dot{\wedge} \xi_{j} \dot{\wedge} H^{2}(\mathbb{D}, E)$ is a closed subspace of $H^{2}\left(\mathbb{D}, \wedge^{j+2} E\right)$, let us consider

$$
\Xi_{j}=\left\{f \in H^{2}(\mathbb{D}, E):\left\langle f(z), \xi_{i}(z)\right\rangle_{E}=0, \text { for } i=0, \ldots, j\right\}
$$

to be the pointwise orthogonal complement of $\xi_{0}, \ldots, \xi_{j}$ in $H^{2}(\mathbb{D}, E)$. Let $\psi \in$ $H^{2}(\mathbb{D}, E)$. We may write $\psi$ as

$$
\psi(z)=\psi(z)-\sum_{i=0}^{j}\left\langle\psi(z), \xi_{i}(z)\right\rangle_{E} \xi_{i}(z)+\sum_{i=0}^{j}\left\langle\psi(z), \xi_{i}(z)\right\rangle_{E} \xi_{i}(z) .
$$

Then, for all $\psi \in H^{2}(\mathbb{D}, E)$ and for almost all $z \in \mathbb{T}$,

$$
\left(\xi_{0} \dot{\wedge} \cdots \dot{\wedge} \xi_{j} \dot{\wedge} \psi\right)(z)=\xi_{0}(z) \wedge \cdots \wedge\left(\psi(z)-\sum_{i=0}^{j}\left\langle\psi(z), \xi_{i}(z)\right\rangle_{E} \xi_{i}(z)\right)
$$

due to the pointwise linear dependence of $\xi_{k}$ and $z \mapsto\left\langle\psi(z), \xi_{k}(z)\right\rangle_{E} \xi_{k}(z)$ almost everywhere on $\mathbb{T}$. 
Notice that $\left(\psi(z)-\sum_{i=0}^{j}\left\langle\psi(z), \xi_{i}(z)\right\rangle_{E} \xi_{i}(z)\right)$ is in $\Xi_{j}$, thus

$$
\xi_{0} \dot{\wedge} \cdots \dot{\wedge} \xi_{j} \dot{\wedge} H^{2}(\mathbb{D}, E) \subset \xi_{0} \dot{\wedge} \cdots \dot{\wedge} \xi_{j} \dot{\wedge} \Xi_{j}
$$

The reverse inclusion holds by the definition of $\Xi_{j}$, hence

$$
\xi_{0} \dot{\wedge} \cdots \dot{\wedge} \xi_{j} \dot{\wedge} H^{2}(\mathbb{D}, E)=\xi_{0} \dot{\wedge} \cdots \dot{\wedge} \xi_{j} \dot{\wedge} \Xi_{j}
$$

Consequently, in order to prove the proposition it suffices to show that $\xi_{0} \dot{\wedge} \cdots \dot{\wedge} \xi_{j} \dot{\wedge} \Xi_{j}$ is a closed subspace of $H^{2}\left(\mathbb{D}, \wedge^{j+2} E\right)$. By Proposition 4.6, $\Xi_{j}$ is a closed subspace of $H^{2}(\mathbb{D}, E)$, being a finite intersection of closed subspaces.

By Lemma 2.15, for any $f \in \Xi_{j}$, we get

$$
\begin{aligned}
& \left\|\xi_{0} \dot{\wedge} \xi_{1} \dot{\wedge} \cdots \dot{\wedge} \xi_{j} \dot{\wedge} f\right\|_{L^{2}\left(\mathbb{T}, \wedge^{j+2} E\right)}^{2} \\
& =\frac{1}{2 \pi} \int_{0}^{2 \pi}\left\|\xi_{0}\left(e^{i \theta}\right) \wedge \xi_{1}\left(e^{i \theta}\right) \wedge \cdots \wedge \xi_{j}\left(e^{i \theta}\right) \wedge f\left(e^{i \theta}\right)\right\|_{\wedge^{j+2} E}^{2} d \theta \\
& =\frac{1}{2 \pi} \int_{0}^{2 \pi}\left\|f\left(e^{i \theta}\right)-\sum_{i=1}^{j}\left\langle f\left(e^{i \theta}\right), \xi_{i}\left(e^{i \theta}\right)\right\rangle \xi_{i}\left(e^{i \theta}\right)\right\|_{E}^{2} d \theta \\
& =\frac{1}{2 \pi} \int_{0}^{2 \pi}\left\|f\left(e^{i \theta}\right)\right\|_{E}^{2} d \theta=\|f\|_{L^{2}(\mathbb{T}, E)}^{2} .
\end{aligned}
$$

Thus

$$
\left(\xi_{0} \dot{\wedge} \xi_{1} \dot{\wedge} \cdots \dot{\wedge} \xi_{j} \dot{\wedge} \cdot\right): \Xi_{j} \rightarrow \xi_{0} \dot{\wedge} \xi_{1} \dot{\wedge} \cdots \dot{\wedge} \xi_{j} \dot{\wedge} \Xi_{j}
$$

is an isometry. Furthermore

$$
\left(\xi_{0} \dot{\wedge} \xi_{1} \dot{\wedge} \cdots \dot{\wedge} \xi_{j} \dot{\wedge} \cdot\right): \Xi_{j} \rightarrow \xi_{0} \dot{\wedge} \xi_{1} \dot{\wedge} \cdots \dot{\wedge} \xi_{j} \dot{\wedge} \Xi_{j}
$$

is a surjective mapping, thus $\Xi_{j}$ and $\xi_{0} \dot{\wedge} \cdots \dot{\wedge} \xi_{j} \dot{\wedge} \Xi_{j}$ are isometrically isomorphic. Therefore, since $\Xi_{j}$ is a closed subspace of $H^{2}(\mathbb{D}, E)$, the space $\xi_{0} \dot{\wedge} \cdots \dot{\wedge} \xi_{j} \dot{\wedge} \Xi_{j}$ is a closed subspace of $H^{2}\left(\mathbb{D}, \wedge^{j+2} E\right)$. Hence

$$
\xi_{0} \dot{\wedge} \cdots \dot{\wedge} \xi_{j} \dot{\wedge} H^{2}(\mathbb{D}, E)
$$

is a closed subspace of $H^{2}\left(\mathbb{D}, \wedge^{j+2} E\right)$.

Proposition 4.8 Let $E$ be a separable Hilbert space, let $F$ be a subspace of $L^{2}(\mathbb{T}, E)$ and let $X$ be a subset of $L^{2}(\mathbb{T}, E)$. The space

$$
\operatorname{POC}(X, F)=\{f \in F: f(z) \perp\{x(z): x \in X\} \text { for almost all } z \in \mathbb{T}\}
$$


is a closed subspace of $F$.

Proof It follows from Proposition 4.6, since $\operatorname{POC}(X, F)$ is an intersection of closed subspaces $V_{x}=\left\{f \in F:\langle f(z), x(z)\rangle_{E}=0\right.$ for almost all $\left.z \in \mathbb{T}\right\}$ over $x \in X$.

Remark 4.9 By the generalized Fatou's Theorem, for $E$ a separable Hilbert space, the space $H^{\infty}(\mathbb{D}, E)$ can be identified with a closed subspace of $L^{\infty}(\mathbb{T}, E)$.

Definition 4.10 Let $E$ be a separable Hilbert space. Let $f \in H^{p}(\mathbb{D}, E)$, for $1 \leq p \leq$ $\infty$. By the generalized Fatou's Theorem (see [8], p. 186), the radial limit

$$
\lim _{r \rightarrow 1} f\left(r e^{i \theta}\right) \underset{\|\cdot\|_{E}}{=} \tilde{f}\left(e^{i \theta}\right)(0<r<1)
$$

exists almost everywhere on $\mathbb{T}$ and defines a function $\tilde{f} \in L^{p}(\mathbb{T}, E)$. The set of points on $\mathbb{T}$ at which the above limit does not exist, will be called the singular set of the function $f$ and will be denoted by $N_{f}$.

Note that the singular sets of functions in $H^{p}(\mathbb{D}, E)$ for $1 \leq p \leq \infty$ are null sets with respect to Lebesgue measure.

Definition 4.11 Let $E$ be a separable Hilbert space. Let $F$ be a subspace of $L^{2}(\mathbb{T}, E)$ and let $X$ be a subset of $L^{2}(\mathbb{T}, E)$. We define the pointwise linear span of $X$ in $F$ to be the set

$$
\operatorname{PLS}(X, F)=\{f \in F: f(z) \in \operatorname{span}\{x(z): x \in X\} \text { for almost all } z \in \mathbb{T}\} .
$$

Recall Definition 4.1, for a separable Hilbert space $E$ and for $\xi \in H^{\infty}(\mathbb{D}, E)$, the pointwise creation operator $C_{\xi}$ is defined by

$$
C_{\xi}: H^{2}(\mathbb{D}, E) \rightarrow H^{2}\left(\mathbb{D}, \wedge^{2} E\right), f \mapsto \xi \dot{\wedge} f,
$$

where

$$
(\xi \dot{\wedge} f)(z)=\xi(z) \wedge f(z) \text { for all } z \in \mathbb{D}
$$

Proposition 4.12 Let $E$ be a separable Hilbert space. For $\xi \in H^{\infty}(\mathbb{D}, E)$,

$$
\operatorname{ker} C_{\xi} \subset \operatorname{PLS}\left(\{\xi\}, H^{2}(\mathbb{D}, E)\right) \text {. }
$$

Proof We have

$$
\begin{aligned}
\operatorname{ker} C_{\xi} & =\left\{f \in H^{2}(\mathbb{D}, E):(\xi \dot{\wedge} f)(z)=0 \text { for all } z \in \mathbb{D}\right\} \\
& =\left\{f \in H^{2}(\mathbb{D}, E): \xi(z) \wedge f(z)=0 \text { for all } z \in \mathbb{D}\right\} \\
& =\left\{f \in H^{2}(\mathbb{D}, E): \xi(z), f(z) \text { are pointwise linearly dependent for all } z \in \mathbb{D}\right\} \\
& \subset \operatorname{PLS}\left(\{\xi\}, H^{2}(\mathbb{D}, E)\right) .
\end{aligned}
$$


Example 4.13 Let $E=\mathbb{C}^{2}$. We can find functions $f, g \in H^{2}(\mathbb{D}, E)$ such that $f \in$ $\operatorname{POC}\left(\{g\}, H^{2}(\mathbb{D}, E)\right)$ but it is false that $\langle f(z), g(z)\rangle_{E}=0$ for all $z \in \mathbb{D}$.

Choose

$$
g(z)=\left(\begin{array}{c}
z \\
z^{2}
\end{array}\right), \quad f(z)=\left(\begin{array}{l}
f_{1}(z) \\
f_{2}(z)
\end{array}\right) \text { for } \quad z \in \mathbb{D} .
$$

Then

$$
f \in \operatorname{POC}\left(\{g\}, \quad H^{2}(\mathbb{D}, E)\right)
$$

is equivalent to

$$
\langle f(z), g(z)\rangle_{E}=0 \text { for almost all } z \in \mathbb{T} \text {. }
$$

The later is equivalent to

$$
\left\langle\left(\begin{array}{c}
z \\
z^{2}
\end{array}\right),\left(\begin{array}{l}
f_{1}(z) \\
f_{2}(z)
\end{array}\right)\right\rangle_{E}=0 \text { for almost all } z \in \mathbb{T}
$$

which holds if and only if

$$
\bar{z} f_{1}(z)+\bar{z}^{2} f_{2}(z)=0 \text { for almost all } z \in \mathbb{T} \text {. }
$$

Equivalently

$$
f_{1}(z)=-\bar{z} f_{2}(z) \text { for almost all } z \in \mathbb{T} \text {, }
$$

which in turn is equivalent to

$$
f(z)=\left(\begin{array}{c}
f_{1}(z) \\
-z f_{1}(z)
\end{array}\right) \quad \text { for almost all } \quad z \in \mathbb{T} .
$$

Now, for $z \in \mathbb{D}$,

$$
\begin{aligned}
\langle f(z), g(z)\rangle_{E} & =\left\langle\left(\begin{array}{c}
f_{1}(z) \\
-z f_{1}(z)
\end{array}\right),\left(\begin{array}{c}
z \\
z^{2}
\end{array}\right)\right\rangle_{E} \\
& =\bar{z} f_{1}(z)-\bar{z}|z|^{2} f_{1}(z) \\
& =\bar{z}\left(1-|z|^{2}\right) f_{1}(z) .
\end{aligned}
$$

So if we take $f_{1}(z)=1, f(z)=\left(\begin{array}{c}1 \\ -z\end{array}\right)$ for $z \in \mathbb{D}$, then $f \in \operatorname{POC}\left(\{g\}, H^{2}(\mathbb{D}, E)\right)$, but $\langle f(z), g(z)\rangle_{E} \neq 0$ for all $z \in \mathbb{D} \backslash\{0\}$.

Thus it is not true in general that $\operatorname{POC}\left(\{g\}, H^{2}(\mathbb{D}, E)\right) \subset\{g\}^{\perp}$. 
Lemma 4.14 Let $E$ be a separable Hilbert space. For $\xi \in H^{\infty}(\mathbb{D}, E)$,

$$
\operatorname{POC}\left(\{\xi\}, H^{2}(\mathbb{D}, E)\right) \subset H^{2}(\mathbb{D}, E) \ominus \operatorname{PLS}\left(\{\xi\}, H^{2}(\mathbb{D}, E)\right)
$$

Proof Let $f \in \operatorname{POC}\left(\{\xi\}, H^{2}(\mathbb{D}, E)\right)$. This is equivalent to $f \in H^{2}(\mathbb{D}, E)$ and

$$
f(z) \perp \xi(z) \text { for all } z \in \mathbb{T} \backslash\left(N_{f} \cup N_{\xi}\right),
$$

where $N_{f}, N_{\xi}$ are the singular sets for the functions $f, \xi$ respectively. This in turn is equivalent to $f \in H^{2}(\mathbb{D}, E)$ and

$$
\langle f(z), \xi(z)\rangle_{E}=0 \text { for all } z \in \mathbb{T} \backslash\left(N_{f} \cup N_{\xi}\right)
$$

The latter implies $f \in H^{2}(\mathbb{D}, E)$ and

$$
\langle f(z), g(z)\rangle_{E}=0 \text { for almost all } z \in \mathbb{T} \text { for all } g \in \operatorname{PLS}\left(\{\xi\}, H^{2}(\mathbb{D}, E)\right) \text {. }
$$

Thus

$$
f \in H^{2}(\mathbb{D}, E) \ominus \operatorname{PLS}\left(\{\xi\}, H^{2}(\mathbb{D}, E)\right) .
$$

Lemma 4.15 Let $E$ and $F$ be separable Hilbert spaces, and let $G \in L^{\infty}(\mathbb{T}, \mathcal{B}(F, E))$. For every $x \in L^{2}(\mathbb{T}, E)$, the function $G x$, defined almost everywhere on $\mathbb{T}$ by

$$
(G x)(z)=G(z)(x(z))
$$

belongs to $L^{2}(\mathbb{T}, E)$.

Proof For almost all $z \in \mathbb{T}$,

$$
\|(G x)(z)\|_{E}=\|G(z) x(z)\|_{E} \leq\|G\|_{L^{\infty}(\mathbb{T}, \mathcal{B}(F, E))}\|x(z)\|_{F} .
$$

Thus

$$
\begin{aligned}
\|G x\|_{L^{2}(\mathbb{T}, E)}^{2} & =\frac{1}{2 \pi} \int_{0}^{2 \pi}\left\|G x\left(e^{i \theta}\right)\right\|_{E}^{2} d \theta \\
& \leq \frac{1}{2 \pi} \int_{0}^{2 \pi}\|G\|_{L^{\infty}(\mathbb{T}, \mathcal{B}(F, E))}^{2}\left\|x\left(e^{i \theta}\right)\right\|_{F}^{2} d \theta \\
& \leq\|G\|_{L^{\infty}(\mathbb{T}, \mathcal{B}(F, E))}^{2}\|x\|_{L^{2}(\mathbb{T}, F)}^{2}<\infty .
\end{aligned}
$$


Definition 4.16 Let $E$ and $F$ be separable Hilbert spaces. Let $P_{+}: L^{2}(\mathbb{T}, E) \rightarrow$ $H^{2}(\mathbb{D}, E)$ be the orthogonal projection operator. Corresponding to any $G \in$ $L^{\infty}(\mathbb{T}, \mathcal{B}(F, E))$ we define the Toeplitz operator with symbol $G$ to be the operator

$$
T_{G}: H^{2}(\mathbb{D}, F) \rightarrow H^{2}(\mathbb{D}, E)
$$

given by

$$
T_{G} x=P_{+}(G x) \text { for any } x \in H^{2}(\mathbb{D}, F) .
$$

Definition 4.17 ([8], p. 190) For a separable Hilbert space $E$, a function $\xi \in$ $H^{\infty}(\mathbb{D}, E)$ will be called inner if for almost every $z \in \mathbb{T}$,

$$
\|\xi(z)\|_{E}=1 .
$$

Theorem 4.18 Let $E$ be a separable Hilbert space, and let $\xi \in H^{\infty}(\mathbb{D}, E)$. Consider the adjoint operator

$$
C_{\xi}^{*}: H^{2}\left(\mathbb{D}, \wedge^{2} E\right) \rightarrow H^{2}(\mathbb{D}, E)
$$

(i) For every $g \in H^{2}(\mathbb{D}, E)$ and $f \in H^{\infty}(\mathbb{D}, E)$,

$$
C_{\xi}^{*}(f \dot{\wedge} g)=P_{+} \alpha_{f},
$$

where $\alpha_{f} \in L^{2}(\mathbb{T}, E)$ is defined by

$$
\alpha_{f}\left(e^{i \theta}\right)=\left\langle f\left(e^{i \theta}\right), \xi\left(e^{i \theta}\right)\right\rangle_{E} g\left(e^{i \theta}\right)-\left\langle g\left(e^{i \theta}\right), \xi\left(e^{i \theta}\right)\right\rangle_{E} f\left(e^{i \theta}\right)
$$

for all $e^{i \theta} \in \mathbb{T} \backslash\left(N_{f} \cup N_{\xi} \cup N_{g}\right)$, and $P_{+}: L^{2}(\mathbb{T}, E) \rightarrow H^{2}(\mathbb{D}, E)$ is the orthogonal projection. Here $N_{f}, N_{g}, N_{\xi}$ are the singular sets of the functions $f, g$ and $\xi$ respectively.

(ii) Let $\xi \in H^{\infty}(\mathbb{D}, E)$ be an inner function. Then, for any $g \in H^{2}(\mathbb{D}, E)$,

$$
C_{\xi}^{*} C_{\xi} g=P_{+} \alpha_{\xi},
$$

where $\alpha_{\xi}=g-\xi \xi^{*} g$. Moreover

$$
C_{\xi}^{*} C_{\xi} g=g-T_{\xi \xi^{*}} g
$$

where $T_{\xi \xi^{*}}: H^{2}(\mathbb{D}, E) \rightarrow H^{2}(\mathbb{D}, E)$ is the Toeplitz operator with symbol $\xi^{*}$. 
Proof (i) By Proposition 3.8, $f \dot{\wedge} g \in H^{2}\left(\mathbb{D}, \wedge^{2} E\right)$. Now, for all $f \in H^{\infty}(\mathbb{D}, E)$, and all $g, h \in H^{2}(\mathbb{D}, E)$, we have

$$
\begin{aligned}
\left\langle C_{\xi}^{*}(f \dot{\wedge} g), h\right\rangle_{H^{2}(\mathbb{D}, E)} & =\left\langle f \dot{\wedge} g, C_{\xi} h\right\rangle_{H^{2}\left(\mathbb{D}, \wedge^{2} E\right)} \\
& =\langle f \dot{\wedge} g, \xi \dot{\wedge} h\rangle_{L^{2}\left(\mathbb{T}, \wedge^{2} E\right)} \\
& =\frac{1}{2 \pi} \int_{0}^{2 \pi}\left\langle f\left(e^{i \theta}\right) \wedge g\left(e^{i \theta}\right), \xi\left(e^{i \theta}\right) \wedge h\left(e^{i \theta}\right)\right\rangle_{\wedge^{2} E} d \theta
\end{aligned}
$$

which, by Proposition 2.14, is equal to

$$
\frac{1}{2 \pi} \int_{0}^{2 \pi} \operatorname{det}\left(\begin{array}{ccc}
\left\langle f\left(e^{i \theta}\right), \xi\left(e^{i \theta}\right)\right\rangle_{E} & \left\langle f\left(e^{i \theta}\right), h\left(e^{i \theta}\right)\right\rangle_{E} \\
\left\langle g\left(e^{i \theta}\right), \xi\left(e^{i \theta}\right)\right\rangle_{E} & \left\langle g\left(e^{i \theta}\right), h\left(e^{i \theta}\right)\right\rangle_{E}
\end{array}\right) d \theta .
$$

The latter in turn is equal to

$$
\begin{aligned}
\frac{1}{2 \pi} & \int_{0}^{2 \pi}\left(\left\langle f\left(e^{i \theta}\right), \xi\left(e^{i \theta}\right)\right\rangle_{E}\left\langle g\left(e^{i \theta}\right), h\left(e^{i \theta}\right)\right\rangle_{E}\right. \\
& \left.-\left\langle f\left(e^{i \theta}\right), h\left(e^{i \theta}\right)\right\rangle_{E}\left\langle g\left(e^{i \theta}\right), \xi\left(e^{i \theta}\right)\right\rangle_{E}\right) d \theta \\
= & \frac{1}{2 \pi} \int_{0}^{2 \pi}\left\langle\left\langle f\left(e^{i \theta}\right), \xi\left(e^{i \theta}\right)\right\rangle_{E} g\left(e^{i \theta}\right)-\left\langle g\left(e^{i \theta}\right), \xi\left(e^{i \theta}\right)\right\rangle_{E} f\left(e^{i \theta}\right), h\left(e^{i \theta}\right)\right\rangle_{E} d \theta
\end{aligned}
$$

which equals

$$
\begin{aligned}
\frac{1}{2 \pi} \int_{0}^{2 \pi}\left\langle\alpha_{f}\left(e^{i \theta}\right), h\left(e^{i \theta}\right)\right\rangle_{E} d \theta & =\left\langle\alpha_{f}, h\right\rangle_{L^{2}(\mathbb{T}, E)} \\
& =\left\langle P_{+}\left(\alpha_{f}\right), h\right\rangle_{H^{2}(\mathbb{D}, E)}
\end{aligned}
$$

where

$$
\alpha_{f}\left(e^{i \theta}\right)=\left\langle f\left(e^{i \theta}\right), \xi\left(e^{i \theta}\right)\right\rangle_{E} g\left(e^{i \theta}\right)-\left\langle g\left(e^{i \theta}\right), \xi\left(e^{i \theta}\right)\right\rangle_{E} f\left(e^{i \theta}\right)
$$

for all $e^{i \theta} \in \mathbb{T} \backslash\left(N_{\xi} \cup N_{f} \cup N_{g}\right)$. Hence $C_{\xi}^{*}(f \dot{\wedge} g)=P_{+} \alpha_{f}$ as required.

Let us show (ii). By Part (i), for all $g, h \in H^{2}(\mathbb{D}, E)$,

$$
\begin{aligned}
\left\langle C_{\xi}^{*} C_{\xi} g, h\right\rangle_{H^{2}(\mathbb{D}, E)} & =\left\langle C_{\xi}^{*}(\xi \dot{\wedge} g), h\right\rangle_{H^{2}(\mathbb{D}, E)} \\
& =\left\langle P_{+}\left(\alpha_{\xi}\right), h\right\rangle_{H^{2}(\mathbb{D}, E)}
\end{aligned}
$$

where

$$
\alpha_{\xi}\left(e^{i \theta}\right)=\left\langle\xi\left(e^{i \theta}\right), \xi\left(e^{i \theta}\right)\right\rangle_{E} g\left(e^{i \theta}\right)-\left\langle g\left(e^{i \theta}\right), \xi\left(e^{i \theta}\right)\right\rangle_{E} \xi\left(e^{i \theta}\right)
$$

for all $e^{i \theta} \in \mathbb{T} \backslash\left(N_{\xi} \cup N_{g} \cup N_{h}\right)$.

Since $\xi$ is inner, $\left\|\xi\left(e^{i \theta}\right)\right\|_{E}=1$ almost everywhere on $\mathbb{T}$, and so, $C_{\xi}^{*} C_{\xi} g=P_{+} \alpha_{\xi}$, where $\alpha_{\xi}=g-\xi \xi^{*} g$. Hence

$$
C_{\xi}^{*} C_{\xi} g=P_{+}\left(g-\xi \xi^{*} g\right)=g-T_{\xi \xi^{*}} g,
$$


where $T_{\xi \xi^{*}} g=P_{+}\left(\xi \xi^{*} g\right)$ is the Toeplitz operator with symbol $\xi^{*}$.

Example 4.19 There exists an inner function $\xi \in H^{\infty}\left(\mathbb{D}, \mathbb{C}^{2}\right)$ such that, for some $h \in H^{2}\left(\mathbb{D}, \mathbb{C}^{2}\right), C_{\xi}^{*} C_{\xi} h$ is not in the pointwise orthogonal complement of $\xi$ in $E$.

Let $\xi \in H^{\infty}\left(\mathbb{D}, \mathbb{C}^{2}\right)$ be an inner function and let $h \in H^{2}\left(\mathbb{D}, \mathbb{C}^{2}\right)$. Let $\xi$, $h$ be given by

$$
\xi(z)=\frac{1}{\sqrt{2}}\left(\begin{array}{l}
1 \\
z
\end{array}\right), \quad h(z)=\left(\begin{array}{l}
1 \\
1
\end{array}\right) \quad \text { for all } z \in \mathbb{D} .
$$

By Theorem 4.18,

$$
C_{\xi}^{*} C_{\xi} h=P_{+} \alpha
$$

where, for all $z \in \mathbb{T}$,

$$
\alpha(z)=\left(\begin{array}{l}
1 \\
1
\end{array}\right)-\left\langle\left(\begin{array}{l}
1 \\
1
\end{array}\right), \frac{1}{\sqrt{2}}\left(\begin{array}{l}
1 \\
z
\end{array}\right)\right\rangle_{\mathbb{C}^{2}} \frac{1}{\sqrt{2}}\left(\begin{array}{l}
1 \\
z
\end{array}\right) .
$$

Calculations yield, for all $z \in \mathbb{T}$,

$$
\begin{array}{r}
\alpha(z)=\left(\begin{array}{l}
1 \\
1
\end{array}\right)-\frac{1}{\sqrt{2}}(1+\bar{z}) \frac{1}{\sqrt{2}}\left(\begin{array}{l}
1 \\
z
\end{array}\right) \\
=\left(\begin{array}{l}
1 \\
1
\end{array}\right)-\frac{1}{2}\left(\begin{array}{c}
1+\bar{z} \\
z(1+\bar{z})
\end{array}\right) \\
=\frac{1}{2}\left(\begin{array}{l}
1-\bar{z} \\
1-z
\end{array}\right) .
\end{array}
$$

Thus

$$
\left(P_{+} \alpha\right)(z)=\frac{1}{2}\left(\begin{array}{c}
1 \\
1-z
\end{array}\right) \text { for all } z \in \mathbb{T} .
$$

The latter expression is not in the pointwise orthogonal complement of $\xi$ in $\mathbb{C}^{2}$, since for all $z \in \mathbb{T}$,

$$
\begin{aligned}
\left\langle\frac{1}{2}\left(\begin{array}{c}
1 \\
1-z
\end{array}\right), \frac{1}{\sqrt{2}}\left(\begin{array}{l}
1 \\
z
\end{array}\right)\right\rangle_{\mathbb{C}^{2}} & =\frac{1}{2 \sqrt{2}}(1 \bar{z})\left(\begin{array}{c}
1 \\
1-z
\end{array}\right) \\
& =\frac{1}{2 \sqrt{2}}\left(1+\bar{z}-|z|^{2}\right)=\frac{1}{2 \sqrt{2}} \bar{z} \neq 0 .
\end{aligned}
$$

Lemma 4.20 Let $E$ be a separable Hilbert space. For every inner function $\xi \in H^{\infty}(\mathbb{D}, E)$

$$
\left\{x \in H^{2}(\mathbb{D}, E):\left\|C_{\xi} x\right\|_{H^{2}\left(\mathbb{D}, \wedge^{2} E\right)}=\|x\|_{H^{2}(\mathbb{D}, E)}\right\}=\operatorname{POC}\left(\{\xi\}, H^{2}(\mathbb{D}, E)\right) .
$$


Proof By Remark 4.4, for every $x \in H^{2}(\mathbb{D}, E),\|x\|_{H^{2}(\mathbb{D}, E)}=\|x\|_{L^{2}(\mathbb{T}, E)}$. Hence

$$
\begin{aligned}
& \left\{x \in H^{2}(\mathbb{D}, E):\left\|C_{\xi} x\right\|_{H^{2}\left(\mathbb{D}, \wedge^{2} E\right)}^{2}=\|x\|_{H^{2}(\mathbb{D}, E)}^{2}\right\} \\
& =\left\{x \in H^{2}(\mathbb{D}, E):\left\|C_{\xi} x\right\|_{L^{2}\left(\mathbb{T}, \wedge^{2} E\right)}^{2}=\|x\|_{L^{2}(\mathbb{T}, E)}^{2}\right\} .
\end{aligned}
$$

By Proposition 2.14, the latter set is equal to

$$
\begin{aligned}
& \left\{x \in H^{2}(\mathbb{D}, E): \frac{1}{2 \pi} \int_{0}^{2 \pi} \operatorname{det}\left(\begin{array}{c}
\left\langle\xi\left(e^{i \theta}\right), \xi\left(e^{i \theta}\right)\right\rangle_{E}\left\langle\xi\left(e^{i \theta}\right), x\left(e^{i \theta}\right)\right\rangle_{E} \\
\left\langle x\left(e^{i \theta}\right), \xi\left(e^{i \theta}\right)\right\rangle_{E}\left\langle x\left(e^{i \theta}\right), x\left(e^{i \theta}\right)\right\rangle_{E}
\end{array}\right) d \theta\right. \\
& \left.=\frac{1}{2 \pi} \int_{0}^{2 \pi}\left\|x\left(e^{i \theta}\right)\right\|_{E}^{2} d \theta\right\} \text {. }
\end{aligned}
$$

Since $\xi$ is inner, $\left\|\xi\left(e^{i \theta}\right)\right\|_{E}=1$ almost everywhere on $\mathbb{T}$, hence the last set is equal to

$$
\begin{aligned}
\{x & \in H^{2}(\mathbb{D}, E): \frac{1}{2 \pi} \int_{0}^{2 \pi}\left(\left\|x\left(e^{i \theta}\right)\right\|_{E}^{2}-\left|\left\langle\xi\left(e^{i \theta}\right), x\left(e^{i \theta}\right)\right\rangle_{E}\right|^{2}\right) d \theta \\
& \left.=\frac{1}{2 \pi} \int_{0}^{2 \pi}\left\|x\left(e^{i \theta}\right)\right\|_{E}^{2} d \theta\right\} \\
& =\left\{x \in H^{2}(\mathbb{D}, E): \frac{1}{2 \pi} \int_{0}^{2 \pi}\left|\left\langle\xi\left(e^{i \theta}\right), x\left(e^{i \theta}\right)\right\rangle_{E}\right|^{2} d \theta=0\right\} \\
& =\left\{x \in H^{2}(\mathbb{D}, E): \xi\left(e^{i \theta}\right) \perp x\left(e^{i \theta}\right) \text { almost everywhere on } \mathbb{T}\right\} \\
& =\operatorname{POC}\left(\{\xi\}, H^{2}(\mathbb{D}, E)\right) .
\end{aligned}
$$

The following example illustrates the fact that, although $C_{\xi}$ is an isometry on $\operatorname{POC}\left\{\xi, H^{2}\left(\mathbb{D}, \mathbb{C}^{2}\right)\right\}$, it is not a partial isometry on $H^{2}\left(\mathbb{D}, \mathbb{C}^{2}\right)$.

Example 4.21 $C_{\xi}^{*} C_{\xi}$ fails to be a projection for some inner function $\xi \in H^{\infty}\left(\mathbb{D}, \mathbb{C}^{2}\right)$. Let us calculate $C_{\xi}^{*} C_{\xi}$ for $\xi(z)=\frac{1}{\sqrt{2}}\left(\begin{array}{l}1 \\ z\end{array}\right), z \in \mathbb{D}$. By Theorem 4.18, for $h \in$ $H^{2}\left(\mathbb{D}, \mathbb{C}^{2}\right)$

$$
C_{\xi}^{*} C_{\xi} h=P_{+} \alpha
$$

where, for all $z \in \mathbb{T}, \alpha(z)$ is given by

$$
\begin{aligned}
\alpha(z) & =h(z)-\langle h(z), \xi(z)\rangle_{E} \xi(z) \\
& =\left(\begin{array}{l}
h_{1}(z) \\
h_{2}(z)
\end{array}\right)-\frac{1}{\sqrt{2}}\left(h_{1}(z)+\bar{z} h_{2}(z)\right) \frac{1}{\sqrt{2}}\left(\begin{array}{l}
1 \\
z
\end{array}\right) \\
& =\left(\begin{array}{l}
h_{1}(z)-\frac{1}{2}\left(h_{1}(z)+\bar{z} h_{2}(z)\right) \\
h_{2}(z)-\frac{1}{2}\left(z h_{1}(z)+h_{2}(z)\right.
\end{array}\right) \\
& =\frac{1}{2}\left(\begin{array}{l}
h_{1}(z)-\bar{z} h_{2}(z) \\
h_{2}(z)-z h_{1}(z)
\end{array}\right) .
\end{aligned}
$$


Thus

$$
P_{+} \alpha=\frac{1}{2}\left(\begin{array}{c}
h_{1}-S^{*} h_{2} \\
-S h_{1}+h_{2}
\end{array}\right)
$$

where $S, S^{*}$ denote the shift and the backward shift operators on $H^{2}(\mathbb{D}, \mathbb{C})$ respectively. Hence

$$
C_{\xi}^{*} C_{\xi}=\frac{1}{2}\left(\begin{array}{cc}
1 & -S^{*} \\
-S & 1
\end{array}\right) .
$$

By Eq. (4.4),

$$
\begin{aligned}
\left(C_{\xi}^{*} C_{\xi}\right)^{2} & =\frac{1}{4}\left(\begin{array}{cc}
1+S^{*} S & -2 S^{*} \\
-2 S & S S^{*}+1
\end{array}\right) \\
& =\frac{1}{4}\left(\begin{array}{cc}
2 & -2 S^{*} \\
-2 S & 2-P_{0}
\end{array}\right) \\
& =\frac{1}{2}\left(\begin{array}{cc}
1 & -S^{*} \\
-S & 1-\frac{1}{2} P_{0}
\end{array}\right) \neq C_{\xi}^{*} C_{\xi},
\end{aligned}
$$

since $S S^{*}=1-P_{0}$, where $P_{0}\left(\sum_{n=0}^{\infty} a_{n} z^{n}\right)=a_{0}$.

Consequently $C_{\xi}^{*} C_{\xi}$ is not a projection and hence $C_{\xi}$ is not a partial isometry on $H^{2}\left(\mathbb{D}, \mathbb{C}^{2}\right)$.

Open Access This article is licensed under a Creative Commons Attribution 4.0 International License, which permits use, sharing, adaptation, distribution and reproduction in any medium or format, as long as you give appropriate credit to the original author(s) and the source, provide a link to the Creative Commons licence, and indicate if changes were made. The images or other third party material in this article are included in the article's Creative Commons licence, unless indicated otherwise in a credit line to the material. If material is not included in the article's Creative Commons licence and your intended use is not permitted by statutory regulation or exceeds the permitted use, you will need to obtain permission directly from the copyright holder. To view a copy of this licence, visit http://creativecommons.org/licenses/by/4.0/.

\section{References}

1. Agler, J., McCarthy, J.E., Young, N.J.: Operator Analysis: Hilbert Space Methods in Complex Analysis, Cambridge Tracts in Mathematics Number 219. Cambridge University Press, Cambridge (2020)

2. Chiotis, D., Lykova, Z. A., Young, N. J.: Exterior Products of Operators and Superoptimal Analytic Approximation, p. 117 (2020). arXiv: 2004.06415 [math.FA]

3. De Pillis, J.: Grassman algebras as Hilbert spaces. J. Algebra 10, 485-500 (1968)

4. Dixmier, J.: Operator Algebras in Hilbert Space (von Neumann Algebras). Èditions Jacques Gabay, Paris (1996). Reprint of the second (1969) edition

5. Greub, W.H.: Multilinear Algebra. Springer, Berlin (1967)

6. Helemskii, A.Y.: The Homology of Banach and Topological Algebras. Translated from the Russian by Alan West. Mathematics and its Applications (Soviet series), vol. 41. Kluwer Academic Publishers Group, Dordrecht (1989) 
7. Horn, R.A., Johnson, C.R.: Matrix Analysis. Cambridge University Press, Cambridge (1985)

8. Nagy, B.S., Foias, C.: Harmonic Analysis of Operators on Hilbert Space. Translated from the French and Revised. North-Holland Publishing Co., Amsterdam (1970)

9. Pavan, V.: Exterior Algebras. Elementary Tribute to Grassmann's Ideas. STE Press, London (2017)

10. Peller, V.V., Young, N.J.: Construction of superoptimal approximants. Math. Control Signals Syst. 8(2), 118-137 (1995)

11. Simon, B.: Trace Ideals and Their Applications. Mathematical Surveys and Monographs, vol. 120, 2nd edn. American Mathematical Society, Providence (2005)

12. Simon, B.: Real Analysis. A Comprehensive Course in Analysis, Part 1. American Mathematical Society, Providence (2015)

13. Winitzki, S.: Linear Algebra via Exterior Products. Published by lulu.com, ISBN 9781409294962 (2010)

Publisher's Note Springer Nature remains neutral with regard to jurisdictional claims in published maps and institutional affiliations. 